\title{
QUASINEUTRAL LIMIT OF THE PRESSURELESS EULER-POISSON EQUATION FOR IONS
}

\author{
BY \\ XUEKE PU (Department of Mathematics, Chongqing University, Chongqing 401331, People's \\ Republic of China) \\ AND \\ BOLING GUO (Institute of Applied Physics and Computational Mathematics, P.O. Box 8009, \\ Beijing, People's Republic of China, 100088)
}

\begin{abstract}
In this paper, we consider the quasineutral limit of the Euler-Poisson equation for cold ions when the Debye length tends to zero. In the cold ion case the Euler-Poisson equation is pressureless and hence fails to be Friedrich symmetrizable, excluding the application of the PsDO energy estimates method of Grenier to obtain uniform estimates independent of $\varepsilon$. To overcome this difficulty, we use $\varepsilon$-weighted norms which combine energy estimates in different levels with weights depending on $\varepsilon$. Finally, that the quasineutral regimes are the compressible Euler equations is proven for well prepared initial data. As a natural extension, we also obtain the zero temperature limit of the Euler-Poisson equation.
\end{abstract}

1. Introduction. In this paper, we consider the Euler-Poisson equation for cold ions in plasma:

$$
(E P)\left\{\begin{array}{l}
\partial_{t} n+\operatorname{div}(n \mathbf{u})=\mathbf{0}, \\
\partial_{t} \mathbf{u}+\mathbf{u} \cdot \nabla \mathbf{u}=-\nabla \phi, \\
\varepsilon \Delta \phi=e^{\phi}-n,
\end{array}\right.
$$

where $n$ is the density of the ions, $\mathbf{u}=\left(u_{1}, \cdots, u_{d}\right)$ is the velocity field and $\phi$ is the electric potential at time $t \in \mathbb{R}^{+}$and position $x \in \mathbb{R}^{d}, d \leq 3$. Here $e^{\phi}$ is the rescaled electron density by the famous Boltzmann relation and $\varepsilon \ll 1$ is a small parameter representing the squared scaled Debye length $\varepsilon=\lambda_{D}^{2} / L^{2}=\epsilon_{0} k_{B} T_{i} / N_{i} e^{2} L^{2}$, where $\lambda_{D}$ is the Debye length, $L$ is the characteristic observation length, $\epsilon_{0}$ is the vacuum permittivity, $\kappa_{B}$ is the

Received August 14, 2014.

2010 Mathematics Subject Classification. Primary 35Q35, 35B25; Secondary 35C20.

Key words and phrases. Euler-Poisson equation, quasineutral limit, compressible Euler equation.

The first author was supported in part by NSFC (11471057) and Natural Science Foundation Project of CQ CSTC (cstc2014jcyjA50020).

E-mail address: xuekepu@cqu.edu.cn

E-mail address: gbl@iapcm.ac.cn 
universal Boltzmann constant, $T_{i}$ and $N_{i}$ are respectively the average temperature and density of ions and $e$ is the fundamental electric charge. For typical plasma applications, the Debye length is very small compared to the characteristic length of physical interest, and it is therefore necessary to consider the limiting system when $\varepsilon \rightarrow 0$. For more physical background on the Euler-Poisson equation or the ion-acoustic plasma, one may refer to [14].

Formally, by letting $\varepsilon \rightarrow 0$, we obtain from the third equation in (1.1) that $\phi=\ln n$, and hence the following compressible Euler system:

$$
(E Q)\left\{\begin{array}{l}
\partial_{t} n+\operatorname{div}(n \mathbf{u})=0 \\
\partial_{t} \mathbf{u}+\mathbf{u} \cdot \nabla \mathbf{u}+\nabla \ln n=0
\end{array}\right.
$$

This limit system (1.2) is a hyperbolic symmetrizable system whose classical result for the existence and uniqueness of sufficiently smooth solutions in a small time interval is available in [17. The system (1.2) has to be supplemented by suitable initial conditions. We shall assume that the plasma is uniform and electrically neutral near infinity, i.e., $n \rightarrow n^{ \pm}$and $\mathbf{u} \rightarrow 0$ as $x \rightarrow \pm \infty$. More precisely, let $\tilde{n}$ be a smooth strictly positive function, constant outside $x \in[-1,+1]$, going to $n^{ \pm}$as $x \rightarrow \infty$. We assume that the initial conditions $\left(n_{0}^{0}, \mathbf{u}_{0}^{0}\right)$ satisfy

$$
\left(n_{0}^{0}-\tilde{n}\right) \in H^{s}\left(\mathbb{R}^{d}\right), \quad \mathbf{u}_{0}^{0} \in \mathbf{H}^{s}\left(\mathbb{R}^{d}\right), \quad n_{0}^{0} \geq \sigma>0,
$$

for some $s>3 / 2$ and some constant $\sigma>0$.

Theorem 1.1. Let $\left(n_{0}^{0}, \mathbf{u}_{0}^{0}\right) \in H^{s^{\prime}} \times H^{s^{\prime}}$ be initial data with $s^{\prime}>\frac{d}{2}+1$ and satisfying (1.3). Then there exists $T>0$, maximal time of existence and a solution $\left(n^{0}, \mathbf{u}^{0}\right)$ of (1.2) on $0 \leq t<T$ with initial data $\left(n_{0}^{0}, \mathbf{u}_{0}^{0}\right)$ such that for every $T^{\prime}<T$,

$$
\left(n^{0}-\tilde{n}, \mathbf{u}^{0}\right) \in\left(C\left([0, T] ; H^{s^{\prime}}\right) \times C\left([0, T] ; \mathbf{H}^{s^{\prime}}\right)\right) \cap\left(C^{1}\left([0, T] ; H^{s^{\prime}-1}\right) \times C^{1}\left([0, T] ; \mathbf{H}^{s^{\prime}-1}\right)\right)
$$

and $T$ depends only on $\left\|\left(n_{0}^{0}-\tilde{n}, \mathbf{u}_{0}^{0}\right)\right\|_{H^{s^{\prime}} \times \mathbf{H}^{s^{\prime}}}$.

Remark 1.2. For the maximal existence time $T$, either $T=\infty$ in the case of global existence or $T<\infty$ and the solution blows up when $t \rightarrow T$ :

$$
\limsup _{t \rightarrow T}\left(\left\|\mathbf{u}^{0}\right\|_{L^{\infty}}+\left\|n^{0}\right\|_{L^{\infty}}+\left\|\frac{1}{n^{0}}\right\|_{L^{\infty}}+\int_{0}^{t}\left(\left\|\partial_{x} \mathbf{u}^{0}\right\|_{L^{\infty}}+\left\|\partial_{x} n^{0}\right\|_{L^{\infty}}\right) d \tau\right)=\infty .
$$

Therefore, we will work on a time interval $\left[0, T^{\prime}\right]$ for $T^{\prime}<T$ (but arbitrarily close to $T$ ) in order to insure $0<\sigma^{\prime}<n^{0}(t, x)<\sigma^{\prime \prime}$ for all $(t, x)$, for some constants $\sigma^{\prime}, \sigma^{\prime \prime}>0$. Here, $\sigma^{\prime}$ may approach 0 as $T^{\prime}$ goes to $T$.

Let us define $\phi^{0}=\ln n^{0}$. The main result in this paper is the following.

Theorem 1.3. Let $s^{\prime} \in \mathbb{N}$ with $s^{\prime}>\left[\frac{d}{2}\right]+2$ be sufficiently large. Let $\left(n_{0}^{0}, \mathbf{u}_{0}^{0}\right) \in H^{s^{\prime}} \times \mathbf{H}^{s^{\prime}}$ and $\left(n^{0}, \mathbf{u}^{0}\right)$ be the solution of the limit system (1.2) on $[0, T)$ with initial data $\left(n_{0}^{0}, \mathbf{u}_{0}^{0}\right)$, given in Theorem 1.1. Then there exist solutions $\left(n^{\varepsilon}(t), \mathbf{u}^{\varepsilon}(t)\right)$ of (1.1) with the same initial data on $\left[0, T^{\varepsilon}\right)$ with $\liminf _{\varepsilon \rightarrow 0} T^{\varepsilon} \geq T$. Moreover, for every $T^{\prime}<T$ and for every $\varepsilon$ small enough, $\varepsilon^{-1}\left(n^{\varepsilon}-n^{0}\right)$ and $\varepsilon^{-1}\left(\mathbf{u}^{\varepsilon}-\mathbf{u}^{0}\right)$ are bounded in $L^{\infty}\left(\left[0, T^{\prime}\right] ; H^{s}\right)$ and $L^{\infty}\left(\left[0, T^{\prime}\right] ; \mathbf{H}^{s}\right)$, respectively, for some $s<s^{\prime}$. 
This theorem will be proved in Section 2. Without essential difficulties, we can show that the same result holds on the torus $\mathbb{T}^{d}$ following the method in the present paper. The details are omitted.

Before proving this theorem, we make some points that stimulated our work in the present paper. The more general isothermal Euler-Poisson equation for ions has the following form:

$$
\left\{\begin{array}{l}
\partial_{t} n+\operatorname{div}(n \mathbf{u})=\mathbf{0}, \\
\partial_{t} \mathbf{u}+\mathbf{u} \cdot \nabla \mathbf{u}+\frac{T_{i}}{n} \nabla n=-\nabla \phi \\
\varepsilon \Delta \phi=e^{\phi}-n,
\end{array}\right.
$$

where $T_{i}>0$ is the ion temperature. When $T_{i}=0$ (compared with the electron temperature), this equation reduces to (1.1) for the cold ions. When $T_{i}>0$, the pressure term introduces a smoothing effect that enables Cordier and Grenier 2] to prove the quasineutral limit as $\varepsilon \rightarrow 0$ by using the pseudodifferential energy estimates method of [6]. It is shown that, under suitable conditions, the solution of (1.5) converges to the following Euler equation as $\varepsilon \rightarrow 0$ :

$$
\left\{\begin{array}{l}
\partial_{t} n+\operatorname{div}(n \mathbf{u})=0, \\
\partial_{t} \mathbf{u}+\mathbf{u} \cdot \nabla \mathbf{u}+\left(T_{i}+1\right) \nabla \ln n=0 .
\end{array}\right.
$$

But their method cannot be applied to the case when $T_{i}=0$, where such a smoothing effect vanishes. The main difference between (1.1) and (1.5) is that (1.5) has the pressure term $T_{i} \nabla \ln n$, which is crucial in proving the quasineutral result of the Euler-Poisson equation (1.5). With this term, the hyperbolic part of (1.5) is Friedrich symmetrizable and the general framework of pseudodifferential operator energy estimates methods of Grenier [6] can be applied. One may refer to 2] for more details of application of this method in treating the quasineutral limit of (1.5). But without the pressure term, as is the case in the present paper, the pseudodifferential energy method cannot apply and no quasineutral limit can be drawn without introducing new techniques, since the hyperbolic part (the equations (1.1a) and (1.1b) ) of (1.1) is not symmetrizable.

Some historic results follow. For the Euler-Poisson equation (1.5), Guo and Pausader [8] constructed global smooth irrotational solutions with small amplitude for this equation with fixed $\varepsilon>0$ and $T_{i}>0$. Very recently, Guo and the first author of the present paper 9] derived the $\mathrm{KdV}$ equation from (1.5) for the full range of $T_{i} \geq 0$, which inspired the first author [19] to derive the Kadomtsev-Petviashvili II equation and the ZakharovKuznetsov equation (ZKE) via the Gardner-Morikawa type transformations. Lannes et al. independently studied the ZKE limit of the Euler-Poisson equation under long wavelength limit [15. Very recently, Han-Kwan also studied the KdV and ZKE limit from the kinetic Vlasov-Poisson system under long wavelength limit. Guo et al. 7] made a breakthrough for the Euler-Maxwell two-fluid system in 3D and proved that irrotational, smooth and localized perturbations of a constant background with small amplitude lead to global smooth solutions. The long wavelength limit of the Euler-Poisson system in [15, 19] could be interpreted as a quasineutral limit of the Euler-Poisson equation to 
the constant solution $(1,0,0)$. However, the quasineutral limit of the present paper concerning solutions $(n, \mathbf{u}, \phi)$ to a general compressible Euler equation (1.6) is new.

For the quasineutral limit of the Euler-Poisson equation, as pointed out above, Cordier and Grenier proved the quasineutral limit from the isothermal Euler-Poisson equation (1.5) to the Euler equation (1.6) in [2]. Wang [24] studied the quasineutral limit of the Euler-Poisson system with and without viscosity. We also would like to remark that Loeper [16] proved quasineutral limit results for the electron Euler-Poisson equation without pressure term and derived the incompressible Euler equation and hence is irrelevant to the present result. Han-Kwan [10] studied the quasineutral limit of the Vlasov-Poisson system with massless electrons, where the author derived the classical isothermal Euler equation for cold ions using the relative entropy method. Very recently, Gerard-Varet et al. studied the quasineutral limit of the system (1.5) in $\mathbb{R}_{+}^{3}$ and boundary layers were constructed. For numerical studies for the pressureless Euler-Poisson equation (1.1), the reader may refer to a recent paper of Degond et al. [3], which analyzes various schemes for the Euler-Poisson-Boltzmann equation. For more results on the quasineutral limit results of the Euler-Poisson equation and related models, one may refer to various recent papers and the references therein; see [1, 4, 5, 12, 18, 20, 22, 24, 25] to list only a few. The difference between [20] and the present paper is that [20] briefly discusses the weak convergence of weak solutions, while the present paper considers the strong convergence of smooth solutions, which requires subtle estimates.

Now, we make a remark on Cordier and Grenier's results on the quasineutral limit for the Euler-Poisson equation for $T_{i}>0$ fixed. The presence of the pressure term $T_{i} \nabla \ln n$ in (1.5) enables them to derive the Euler equation as Debye length goes to zero. But their estimates are not uniform in $T_{i}>0$, because the smoothing effects of the pressure term vanish as $T_{i} \rightarrow 0$. One may refer to Guo-Pu [9] for a clear study of why these smoothing effects vanish. But by a similar method to the one we use to prove Theorem 1.3 in Section 2, we can indeed give uniform estimates in $T_{i}>0$ and hence improve their results. This would yield the zero temperature limit as $T_{i} \rightarrow 0$ stated in Theorem 1.4 below. Let $\left(n^{\varepsilon, T_{i}}, \mathbf{u}^{\varepsilon, T_{i}}, \phi^{\varepsilon, T_{i}}\right)$ be a solution of (1.5) and $\left(n^{0, T_{i}}, \mathbf{u}^{0, T_{i}}, \phi^{0, T_{i}}\right)$ be a solution of (1.6) with the same initial data. We let

$$
n^{\varepsilon, T_{i}}=n^{0, T_{i}}+\varepsilon n^{1, T_{i}}, \quad \mathbf{u}^{\varepsilon, T_{i}}=\mathbf{u}^{0, T_{i}}+\varepsilon \mathbf{u}^{1, T_{i}}, \quad \phi^{\varepsilon, T_{i}}=\phi^{0, T_{i}}+\varepsilon \phi^{1, T_{i}} .
$$

Here $\phi^{\varepsilon, T_{i}}$ and $n^{\varepsilon, T_{i}}$ satisfy the Poisson equation (1.5c), and indeed $\phi^{\varepsilon, T_{i}}$ can be solved via $\phi^{\varepsilon, T_{i}}=\phi^{\varepsilon, T_{i}}\left[n^{\varepsilon, T_{i}}\right]$ and $\phi^{0, T_{i}}=\ln n^{0, T_{i}}$. We will prove the following.

Theorem 1.4. Let $\left(n^{0, T_{i}}, \mathbf{u}^{0, T_{i}}, \phi^{0, T_{i}}\right)$ be a solution of (1.6) on $[0, T)$ with initial data $\left(n_{0}, \mathbf{u}_{0}\right) \in H^{s^{\prime}}$ with $s^{\prime}$ sufficiently large. There exist solutions $\left(n^{\varepsilon, T_{i}}, \mathbf{u}^{\varepsilon, T_{i}}, \phi^{\varepsilon, T_{i}}\right)$ of (1.5) with the same initial data on $\left[0, T^{\varepsilon, T_{i}}\right)$ with $\liminf _{\varepsilon, T_{i} \rightarrow 0} T^{\varepsilon, T_{i}} \geq T$. Moreover, for every $T^{\prime}<T$ and for $\varepsilon, T_{i}$ small enough, $\left(n^{1, T_{i}}, \mathbf{u}^{1, T_{i}}\right)$ are bounded in $L^{\infty}\left(\left[0, T^{\prime}\right] ; H^{s}\right)$ for some $s<s^{\prime}$.

By such a result, we can indeed derive the Euler equation (1.2) from (1.5) by first letting $\varepsilon \rightarrow 0$ and then letting $T_{i} \rightarrow 0$. The limit $T_{i} \rightarrow 0$ is usually known as the cold ion limit. Furthermore, $T_{i}>0$ and $\varepsilon>0$ don't depend on each other. They can go to zero independently. 
The next section is devoted to the proof of Theorem 1.3 For this purpose, we write the solution of (1.1) as $n^{\varepsilon}=n^{0}+\varepsilon n^{1}$ and $\mathbf{u}^{\varepsilon}=\mathbf{u}^{0}+\varepsilon \mathbf{u}^{1}$ and consider the remainder system $\left(R_{\varepsilon}\right)$ of $n^{1}$ and $\mathbf{u}^{1}$. The main idea is then to show that $\left(n^{1}, \mathbf{u}^{1}\right)$ is uniformly bounded in $H^{s} \times \mathbf{H}^{s}$ when $\varepsilon \rightarrow 0$. To overcome the difficulty of non-symmetrizability of (1.1), we introduce some triple norm ||$|\cdot|||_{\varepsilon, s}$,

$$
\begin{aligned}
& \left.\left\|\mathbf{u}^{1}\right\|\right|_{\varepsilon, s} ^{2}=\left\|\mathbf{u}^{1}\right\|_{H^{s}}^{2}+\varepsilon\left\|\nabla \mathbf{u}^{1}\right\|_{H^{s}}^{2}, \\
& \left.\left\|\phi^{1}\right\|\right|_{\varepsilon, s} ^{2}=\left\|\phi^{1}\right\|_{H^{s}}^{2}+\varepsilon\left\|\phi^{1}\right\|_{H^{s}}^{2}+\varepsilon^{2}\left\|\Delta \phi^{1}\right\|_{H^{s}}^{2},
\end{aligned}
$$

and then show that $\left\|\left|\left(\mathbf{u}^{1}, \phi^{1}\right)\right|\right\|_{\varepsilon, s}$ is uniformly bounded on some time interval independent of $\varepsilon$. The main novelty of the proof is then to combine the $s$-order energy estimates with the $(s+1)$-order energy estimates with weights 1 and $\varepsilon$. By such a combination, we obtain some Gronwall type inequality for $\left\|\left|\left(\mathbf{u}^{1}, \phi^{1}\right)\right|\right\|_{\varepsilon, s}$, which enables us to obtain uniform estimates independent of $\varepsilon$. The estimates of $\left\|n^{1}\right\|_{H^{s}}$ are obtained through some elliptic estimates from the Poisson equation (1.1c) in Section 2.2. This method was successfully employed in our previous paper to handle the long wavelength limit of the Euler-Poisson system to the KdV equation in [9] and could be potentially useful in treating the quasineutral limit for the pressureless electron Euler-Poisson equations.

Theorem 1.4 is proven in Section 3 in the same spirit as the proof of Theorem 1.3 .

We introduce some notation. We let $L^{p}$ denote the usual Lebesgue space of $p$-th integrable functions normed by $\|\cdot\|_{L^{p}}$. When $p=2$, we usually use $\|\cdot\|$ instead of $\|\cdot\|_{L^{2}}$. The Sobolev space $H^{s}, s \in \mathbb{Z}, s \geq 0$ is defined as $H^{s}\left(\mathbb{R}^{d}\right)=\left\{f(x): \sum_{|\alpha| \leq s}\left\|\partial^{\alpha} f\right\|^{2}<\infty\right\}$,

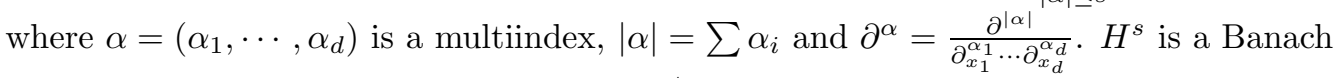
space with norm $\|f\|_{H^{s}}=\left(\sum_{|\alpha| \leq s}\left\|\partial^{\alpha} f\right\|^{2}\right)^{1 / 2}$. For definiteness, we will restrict ourselves to the physical space dimensions $d \leq 3$ in this paper.

2. Proof of Theorem 1.3. The purpose of this section is to prove Theorem 1.3, Let $\left(n^{\varepsilon}, \mathbf{u}^{\varepsilon}, \phi^{\varepsilon}\right)$ satisfy the Euler-Poisson equation (1.1) and $\left(n^{0}, \mathbf{u}^{0}, \phi^{0}\right)$ be a sufficiently smooth solution of the Euler equation (1.2). We let

$$
n^{\varepsilon}=n^{0}+\varepsilon n^{1}, \quad \mathbf{u}^{\varepsilon}=\mathbf{u}^{0}+\varepsilon \mathbf{u}^{1}, \quad \phi^{\varepsilon}=\phi^{0}+\varepsilon \phi^{1} .
$$

Here $\phi^{\varepsilon}$ and $n^{\varepsilon}$ satisfy the Poisson equation (1.1c), and indeed $\phi^{\varepsilon}$ can be solved via $\phi^{\varepsilon}=\phi^{\varepsilon}\left[n^{\varepsilon}\right]$ and $\phi^{0}=\ln n^{0}$. Then $\left(n^{1}, \mathbf{u}^{1}, \phi^{1}\right)$ satisfy the remainder system $\left(R_{\varepsilon}\right)$ :

$$
\left(R_{\varepsilon}\right)\left\{\begin{array}{l}
\partial_{t} n^{1}+\nabla \cdot\left(n^{0} \mathbf{u}^{1}+\mathbf{u}^{0} n^{1}\right)+\varepsilon \nabla \cdot\left(n^{1} \mathbf{u}^{1}\right)=0 \\
\partial_{t} \mathbf{u}^{1}+\mathbf{u}^{0} \cdot \nabla \mathbf{u}^{1}+\mathbf{u}^{1} \cdot \nabla \mathbf{u}^{0}+\varepsilon \mathbf{u}^{1} \cdot \nabla \mathbf{u}^{1}=-\nabla \phi^{1} \\
-\varepsilon \Delta \phi^{1}=\Delta \phi^{0}+n^{1}-n^{0} \phi^{1}+\sqrt{\varepsilon} R^{1}
\end{array}\right.
$$

where

$$
R^{1}=\varepsilon^{-3 / 2}\left(n^{0}+\varepsilon n^{0} \phi^{1}-e^{\phi^{0}+\varepsilon \phi^{1}}\right) .
$$

To prove Theorem 1.3, we need only to derive some uniform bound for the remainder equation (2.2). To slightly simplify the presentation, we assume that (2.2) has smooth solutions in a small time $T_{\varepsilon}$ dependent on $\varepsilon$. Let $\tilde{C}$ be a constant to be determined later, 
much larger than the bound of $\left\|\left(n_{0}^{1}, \mathbf{u}_{0}^{1}\right)\right\|_{s}$, such that on $\left[0, T_{\varepsilon}\right]$,

$$
\sup _{\left[0, T_{\varepsilon}\right]}\left\|\left(n^{1}, \mathbf{u}^{1}, \phi^{1}\right)\right\|_{H^{s}} \leq \tilde{C} .
$$

We will prove that $T_{\varepsilon}>T$ as $\varepsilon \rightarrow 0$ for some $T>0$. Recalling the expressions for $n$ and $\mathbf{u}$ in (2.1), we immediately know that there exists some $\varepsilon_{1}=\varepsilon_{1}(\tilde{C})>0$ such that on $\left[0, T_{\varepsilon}\right]$,

$$
\sigma^{\prime} / 2<n^{\varepsilon}<2 \sigma^{\prime \prime}, \quad\left|\mathbf{u}^{\varepsilon}\right| \leq 1 / 2
$$

for all $0<\varepsilon<\varepsilon_{1}$.

2.1. Estimates for $R_{1}$. We first bound $R^{1}$ in terms of $\phi^{1}$. More precisely, we have the following.

Lemma 2.1. Let $\left(n^{0}, \mathbf{u}^{0}, \phi^{0}\right)$ be a sufficiently smooth solution of (1.2) by Theorem 1.1, Then for the remainder term (2.3), we have on $\left[0, T_{\varepsilon}\right]$,

$$
\begin{aligned}
\left\|R^{1}\right\|_{H^{k}} & \leq C(\sqrt{\varepsilon} \tilde{C})\left\|\phi^{1}\right\|_{H^{k}} \quad \text { and } \\
\left\|\partial_{t} R^{1}\right\|_{H^{k}} & \leq C(\sqrt{\varepsilon} \tilde{C})\left(\left\|\phi^{1}\right\|_{H^{k}}+\left\|\partial_{t} \phi^{1}\right\|_{H^{k}}\right), \quad \forall k \geq 0 .
\end{aligned}
$$

In particular, there exists some $\varepsilon_{1}>0$ and $C_{1}=C(1)$ such that

$$
\begin{aligned}
\left\|R^{1}\right\|_{H^{k}} & \leq C_{1}\left\|\phi^{1}\right\|_{H^{k}} \quad \text { and } \\
\left\|\partial_{t} R^{1}\right\|_{H^{k}} & \leq C_{1}\left(\left\|\phi^{1}\right\|_{H^{k}}+\left\|\partial_{t} \phi^{1}\right\|_{H^{k}}\right), \quad \forall k \geq 0,
\end{aligned}
$$

for all $0<\varepsilon<\varepsilon_{1}$ and $t \in\left[0, T_{\varepsilon}\right]$.

Proof. From the Taylor expansion in the integral form, we have

$$
R^{1}=\varepsilon^{1 / 2} e^{\phi^{0}} \int_{0}^{1} e^{\theta \varepsilon \phi^{1}}(1-\theta) d \theta\left(\phi^{1}\right)^{2} .
$$

By taking $L^{2}$ norm, we have

$$
\left\|R^{1}\right\| \leq \sqrt{\varepsilon}\left\|e^{\phi^{0}}\right\|_{L^{\infty}} e^{\varepsilon\left\|\phi^{1}\right\|_{L^{\infty}}}\left\|\phi^{1}\right\|_{L^{2}}\left\|\phi^{1}\right\|_{L^{\infty}} .
$$

From the continuity assumption (2.4), we have $\left\|\phi^{1}\right\|_{L^{\infty}} \leq C \tilde{C}$ on $\left[0, T_{\varepsilon}\right]$ and hence

$$
\left\|R^{1}\right\|_{L^{2}} \leq C(\sqrt{\varepsilon} \tilde{C})\left\|\phi^{1}\right\|_{L^{2}}, \quad \forall t \in\left[0, T_{\varepsilon}\right]
$$

By applying $\partial^{\alpha}$ with $|\alpha|=k, k \geq 1$ integers, similar estimates yield

$$
\left\|R^{1}\right\|_{H^{k}} \leq C(\sqrt{\varepsilon} \tilde{C})\left\|\phi^{1}\right\|_{H^{k}} .
$$

Taking $\partial_{t}$ to $R^{1}$ and then taking the $H^{k}$ norm, we obtain

$$
\left\|\partial_{t} R^{1}\right\|_{H^{k}} \leq C(\sqrt{\varepsilon} \tilde{C})\left(\left\|\phi^{1}\right\|_{H^{k}}+\left\|\partial_{t} \phi^{1}\right\|_{H^{k}}\right) .
$$

Finally, choosing $\varepsilon_{1}=(1 / \tilde{C})^{2}$ yields (2.7). 
2.2. Elliptical estimates. The following lemmas provide useful estimates between $n^{1}$, $\mathbf{u}^{1}$ and $\phi^{1}$. These will be used widely in the uniform estimates in the next subsection.

Lemma 2.2. Let $\left(n^{1}, \mathbf{u}^{1}, \phi^{1}\right)$ be a smooth solution for the remainder system $\left(R_{\varepsilon}\right)$ and $\alpha$ a multiindex. There exist $\varepsilon_{1}$ and $C$ such that for any $0<\varepsilon<\varepsilon_{1}$ and any multiindices $\alpha$ with $|\alpha|=k \geq 0$, there hold

$$
\begin{aligned}
& \left\|\partial_{x}^{\alpha} n^{1}\right\|^{2} \leq C+C\left\|\phi^{1}\right\|_{H^{k}}^{2}+C \varepsilon^{2}\left\|\Delta \phi^{1}\right\|_{H^{k}}^{2} \quad \text { and } \\
& \left\|\partial_{x}^{\alpha} \phi^{1}\right\|^{2}+\varepsilon\left\|\partial_{x}^{\alpha} \nabla \phi^{1}\right\|+\varepsilon^{2}\left\|\Delta \partial_{x}^{\alpha} \phi^{1}\right\|^{2} \leq C+C\left\|\partial_{x}^{\alpha} n^{1}\right\|^{2}
\end{aligned}
$$

on the interval $\left[0, T_{\varepsilon}\right]$.

Proof. Taking the $L^{2}$ inner product of (2.2c) with $\phi^{1}$ and then integrating by parts yield

$$
\varepsilon\left\|\nabla \phi^{1}\right\|^{2}+\int n^{0}\left|\phi^{1}\right|^{2}=\int \phi^{1} \Delta \phi^{0}+\int n^{1} \phi^{1}+\sqrt{\varepsilon} \int \phi^{1} R^{1} .
$$

Hereafter, $\int=\int_{\mathbb{R}^{d}} \cdots d x$. As $n^{0}>\sigma^{\prime}>0$ for $t \leq T^{\prime}<T$, we obtain by Young's inequality

$$
\varepsilon\left\|\nabla \phi^{1}\right\|^{2}+\sigma^{\prime}\left\|\phi^{1}\right\|^{2} \leq \frac{\sigma^{\prime}}{4}\left\|\phi^{1}\right\|^{2}+\frac{4 C}{\sigma^{\prime}}\left(\left\|\Delta \phi^{0}\right\|^{2}+\left\|n^{1}\right\|^{2}+\varepsilon\left\|R^{1}\right\|^{2}\right) .
$$

From Lemma 2.1, there exists $\varepsilon_{1}>0$ such that $\left\|R^{1}\right\| \leq C_{1}\left\|\phi^{1}\right\|$. Then by choosing a new smaller $\varepsilon_{1}$ such that $\varepsilon_{1} \leq \frac{\sigma^{\prime 2}}{16 C C_{1}}$, we then have for any $0<\varepsilon<\varepsilon_{1}$ that

$$
\varepsilon\left\|\nabla \phi^{1}\right\|^{2}+\sigma^{\prime}\left\|\phi^{1}\right\|^{2} \leq \frac{C}{\sigma^{\prime}}\left(1+\left\|n^{1}\right\|^{2}\right) .
$$

Similarly, by taking the $L^{2}$ inner product of (2.2c) with $\varepsilon \Delta \phi^{1}$ and integrating by parts, we obtain that

$$
\begin{aligned}
\varepsilon^{2}\left\|\Delta \phi^{1}\right\|^{2} & +\varepsilon \int n^{0}\left|\nabla \phi^{1}\right|^{2}=-\varepsilon \int \Delta \phi^{0} \Delta \phi^{1}+\varepsilon \int n^{1} \Delta \phi^{1} \\
& -\varepsilon \int \nabla n^{0} \phi^{1} \nabla \phi^{1}+\varepsilon^{3 / 2} \int R^{1} \phi^{1} \\
\leq & \frac{\varepsilon^{2}}{2}\left\|\Delta \phi^{1}\right\|^{2}+4\left(\left\|\Delta \phi^{0}\right\|^{2}+\left\|n^{1}\right\|^{2}+\varepsilon\left\|R^{1}\right\|^{2}+\varepsilon\left\|\phi^{1}\right\|^{2}+\varepsilon\left\|\nabla \phi^{1}\right\|^{2}\right),
\end{aligned}
$$

which yields for any $0<\varepsilon<\varepsilon_{1}$ for some $\varepsilon_{1}>0$ that

$$
\varepsilon^{2}\left\|\Delta \phi^{1}\right\|^{2}+\varepsilon \sigma^{\prime}\left\|\nabla \phi^{1}\right\|^{2} \leq C\left(1+\left\|n^{1}\right\|^{2}\right),
$$

as $n^{0}>\sigma^{\prime}>0$ for $t \leq T^{\prime}<T$, where the constant $C$ depends on $\sigma^{\prime}$. By combining (2.8) and (2.9) we easily obtain that for any $0<\varepsilon<\varepsilon_{1}$,

$$
\varepsilon^{2}\left\|\Delta \phi^{1}\right\|^{2}+\varepsilon \sigma^{\prime}\left\|\nabla \phi^{1}\right\|^{2}+\left\|\phi^{1}\right\|^{2} \leq C\left(1+\left\|n^{1}\right\|^{2}\right),
$$

for some constant $C$ depending on $\sigma^{\prime}$. On the other hand, by taking the $L^{2}$ norm of (2.2C), we obtain that for any $0<\varepsilon<\varepsilon_{1}$,

$$
\begin{aligned}
\left\|n^{1}\right\|^{2} & \leq \varepsilon^{2}\left\|\Delta \phi^{1}\right\|^{2}+\left\|n^{0}\right\|_{L^{\infty}}^{2}\left\|\phi^{1}\right\|^{2}+\left\|\Delta \phi^{0}\right\|^{2}+\varepsilon\left\|R^{1}\right\|^{2} \\
& \leq C\left(1+\left\|\phi^{1}\right\|^{2}+\varepsilon^{2}\left\|\Delta \phi^{1}\right\|^{2}\right),
\end{aligned}
$$


thanks again to Lemma 2.1, where $C$ depends on $\sigma^{\prime \prime}$ and $C_{1}$. Therefore, we finish the proof when $k=0$. Higher order estimates can be handled similarly, and we omit further details.

Lemma 2.3. Let $\left(n^{1}, \mathbf{u}^{1}, \phi^{1}\right)$ be a smooth solution for the remainder system $\left(R_{\varepsilon}\right)$ and $\alpha$ an integer. There exist $\varepsilon_{1}$ and $C$ such that for any $0<\varepsilon<\varepsilon_{1}$ and any multiindices $\alpha$ with $|\alpha|=k$, there holds

$$
\left\|\partial^{\alpha} \partial_{t} n^{1}\right\|^{2} \leq C\left(1+\left\|\mathbf{u}^{1}\right\|_{\mathbf{H}^{k+1}}^{2}+\left\|\phi^{1}\right\|_{H^{k+1}}^{2}+\varepsilon^{2}\left\|\Delta \phi^{1}\right\|_{H^{k+1}}^{2}\right)
$$

on the time interval $\left[0, T_{\varepsilon}\right]$.

Proof. We take the $L^{2}$ norm of (2.2a) to obtain

$$
\begin{aligned}
\left\|\partial_{t} n^{1}\right\|^{2} & \leq C\left(\mathbf{u}^{1}\left\|_{\mathbf{H}^{1}}^{2}+\right\| n^{1} \|_{H^{1}}^{2}\right)+\varepsilon^{2}\left(\left\|n^{1}\right\|_{L^{\infty}}^{2}\left\|\nabla \mathbf{u}^{1}\right\|^{2}+\left\|\mathbf{u}^{1}\right\|_{L^{\infty}}^{2}\left\|\nabla n^{1}\right\|^{2}\right) \\
& \leq C\left(1+\varepsilon^{2}\left(\left\|n^{1}\right\|_{L^{\infty}}^{2}+\left\|\mathbf{u}^{1}\right\|_{L^{\infty}}^{2}\right)\right)\left(\left\|\mathbf{u}^{1}\right\|_{\mathbf{H}^{1}}^{2}+\left\|n^{1}\right\|_{H^{1}}^{2}\right),
\end{aligned}
$$

for some constant $C$ depending on $\left(n^{0}, \mathbf{u}^{0}\right)$. By the continuity assumption (2.4) and Lemma 2.2, we have

$$
\begin{aligned}
\left\|\partial_{t} n^{1}\right\|^{2} & \leq C\left(1+\varepsilon^{2} \tilde{C}\right)\left(1+\left\|\mathbf{u}^{1}\right\|_{\mathbf{H}^{1}}^{2}+\left\|\phi^{1}\right\|_{H^{1}}^{2}+\varepsilon^{2}\left\|\Delta \phi^{1}\right\|_{H^{1}}^{2}\right), \\
& \leq C\left(1+\left\|\mathbf{u}^{1}\right\|_{\mathbf{H}^{1}}^{2}+\left\|\phi^{1}\right\|_{H^{1}}^{2}+\varepsilon^{2}\left\|\Delta \phi^{1}\right\|_{H^{1}}^{2}\right)
\end{aligned}
$$

for any $0<\varepsilon<\varepsilon_{1}$, for some $\varepsilon_{1}>0$.

Higher order inequalities are proved similarly. Taking $\partial^{\alpha}$ with $|\alpha|=k \geq 1$ to the equation (2.2a and then taking the $L^{2}$ norm, we obtain

$$
\begin{aligned}
\left\|\partial^{\alpha} \partial_{t} n^{1}\right\|^{2} & \leq C\left(\mathbf{u}^{1}\left\|_{\mathbf{H}^{k+1}}^{2}+\right\| n^{1} \|_{H^{k+1}}^{2}\right)+\varepsilon^{2}\left(\left\|n^{1}\right\|_{L^{\infty}}^{2}\left\|\partial^{\alpha} \nabla \mathbf{u}^{1}\right\|^{2}+\left\|\mathbf{u}^{1}\right\|_{L^{\infty}}^{2}\left\|\partial^{\alpha} \nabla n^{1}\right\|^{2}\right) \\
& \leq C\left(1+\varepsilon^{2}\left(\left\|n^{1}\right\|_{L^{\infty}}^{2}+\left\|\mathbf{u}^{1}\right\|_{L^{\infty}}^{2}\right)\right)\left(\left\|\mathbf{u}^{1}\right\|_{\mathbf{H}^{k+1}}^{2}+\left\|n^{1}\right\|_{H^{k+1}}^{2}\right) \\
& \leq C\left(1+\left\|\mathbf{u}^{1}\right\|_{\mathbf{H}^{k+1}}^{2}+\left\|\phi^{1}\right\|_{H^{k+1}}^{2}+\varepsilon^{2}\left\|\Delta \phi^{1}\right\|_{H^{k+1}}^{2}\right),
\end{aligned}
$$

for any $0<\varepsilon<\varepsilon_{1}$, for some $\varepsilon_{1}>0$, where we have used the multiplicative estimates in Lemma A.1

Lemma 2.4. Let $\left(n^{1}, \mathbf{u}^{1}, \phi^{1}\right)$ be a smooth solution for the remainder system $\left(R_{\varepsilon}\right)$ and $\alpha$ an integer. There exist $\varepsilon_{1}$ and $C$ such that for any $0<\varepsilon<\varepsilon_{1}$ and any multiindices $\alpha$ with $|\alpha|=k$,

$$
\left\|\partial^{\alpha} \partial_{t} \phi^{1}\right\|^{2}+\varepsilon\left\|\partial^{\alpha} \nabla \partial_{t} \phi^{1}\right\|^{2}+\varepsilon^{2}\left\|\partial^{\alpha} \Delta \partial_{t} \phi^{1}\right\|^{2} \leq C\left(1+\left\|\partial^{\alpha} \partial_{t} n^{1}\right\|^{2}+\left\|\phi^{1}\right\|_{H^{k}}^{2}\right)
$$

on the time interval $\left[0, T_{\varepsilon}\right]$.

Proof. Taking $\partial_{t}$ of (2.2c), we obtain

$$
-\varepsilon \Delta \partial_{t} \phi^{1}=\Delta \partial_{t} \phi^{0}+\partial_{t} n^{1}-\partial_{t}\left(n^{0} \phi^{1}\right)+\sqrt{\varepsilon} \partial_{t} R^{1} .
$$

Taking the $L^{2}$ inner product with $\partial_{t} \phi^{1}$ and then integrating by parts, we obtain

$$
\varepsilon\left\|\nabla \partial_{t} \phi^{1}\right\|^{2}+\int n^{0}\left|\partial_{t} \phi^{1}\right|^{2}=\int \partial_{t} \phi^{1}\left(\Delta \partial_{t} \phi^{0}-\partial_{t} n^{0} \phi^{1}+\partial_{t} n^{1}+\sqrt{\varepsilon} \partial_{t} R^{1}\right) .
$$


As $n^{0}>\sigma^{\prime}>0$ for $t \leq T^{\prime}<T$, we have by Hölder inequality

$$
\begin{aligned}
\varepsilon\left\|\nabla \partial_{t} \phi^{1}\right\|^{2} & +\sigma^{\prime}\left\|\partial_{t} \phi^{1}\right\|^{2} \leq \frac{\sigma^{\prime}}{4}\left\|\partial_{t} \phi^{1}\right\|^{2} \\
& +\frac{4}{\sigma^{\prime}}\left(\left\|\Delta \partial_{t} \phi^{0}\right\|^{2}+\left\|\partial_{t} n^{0}\right\|_{L^{\infty}}^{2}\left\|\phi^{1}\right\|^{2}+\left\|\partial_{t} n^{1}\right\|^{2}+\varepsilon\left\|\partial_{t} R^{1}\right\|^{2}\right) \\
\leq & \frac{\sigma^{\prime}}{4}\left\|\partial_{t} \phi^{1}\right\|^{2}+\frac{4}{\sigma^{\prime}}\left(C+C\left\|\phi^{1}\right\|^{2}+\left\|\partial_{t} n^{1}\right\|^{2}+\varepsilon\left\|\partial_{t} R^{1}\right\|^{2}\right) .
\end{aligned}
$$

By choosing a small $\varepsilon_{1}>0$ such that $16 C_{1} \varepsilon_{1} \leq \sigma^{\prime 2}$, we then have for any $0<\varepsilon<\varepsilon_{1}$ that

$$
\varepsilon\left\|\nabla \partial_{t} \phi^{1}\right\|^{2}+\sigma^{\prime}\left\|\partial_{t} \phi^{1}\right\|^{2} \leq \frac{C}{\sigma^{\prime}}\left(1+\left\|\phi^{1}\right\|^{2}+\left\|\partial_{t} n^{1}\right\|^{2}\right),
$$

thanks to Lemma 2.1. Similarly, by taking the inner product with $\varepsilon \Delta \partial_{t} \phi^{1}$, we obtain

$$
\varepsilon^{2}\left\|\Delta \partial_{t} \phi^{1}\right\|^{2}+\sigma^{\prime} \varepsilon\left\|\partial_{t} \phi^{1}\right\|^{2} \leq \frac{C}{\sigma^{\prime}}\left(1+\left\|\phi^{1}\right\|^{2}+\left\|\partial_{t} n^{1}\right\|^{2}\right) .
$$

Adding them together, we obtain that for any $0<\varepsilon<\varepsilon_{1}$ for some $\varepsilon_{1}>0$,

$$
\left\|\partial_{t} \phi^{1}\right\|^{2}+\varepsilon\left\|\nabla \partial_{t} \phi\right\|^{2}+\varepsilon^{2}\left\|\Delta \partial_{t} \phi^{1}\right\|^{2} \leq C\left(1+\left\|\partial_{t} n^{1}\right\|_{H^{k}}^{2}+\left\|\phi^{1}\right\|_{H^{k}}^{2}\right),
$$

for some constant $C$ depending on $\sigma^{\prime}$. Higher order estimates can be treated similarly, and we obtain for any $\alpha$ with $|\alpha|=k$ that

$$
\left\|\partial_{t} \partial^{\alpha} \phi^{1}\right\|^{2}+\varepsilon\left\|\partial_{t} \partial^{\alpha} \nabla \phi\right\|^{2}+\varepsilon^{2}\left\|\partial_{t} \partial^{\alpha} \Delta \phi^{1}\right\|^{2} \leq C\left(1+\left\|\partial_{t} n^{1}\right\|_{H^{k}}^{2}+\left\|\phi^{1}\right\|_{H^{k}}^{2}\right),
$$

for some constant $C$ depending on $\sigma^{\prime}$.

By recalling Lemma 2.3, we have the following.

Corollary 2.5. Let $\left(n^{1}, \mathbf{u}^{1}, \phi^{1}\right)$ be a smooth solution for the remainder system $\left(R_{\varepsilon}\right)$ and $\alpha$ an integer. There exist $\varepsilon_{1}$ and $C$ such that for any $0<\varepsilon<\varepsilon_{1}$ and any multiindices $\alpha$ with $|\alpha|=k$,

$$
\begin{aligned}
\left\|\partial^{\alpha} \partial_{t} \phi^{1}\right\|^{2} & +\varepsilon\left\|\partial^{\alpha} \nabla \partial_{t} \phi^{1}\right\|^{2}+\varepsilon^{2}\left\|\partial^{\alpha} \Delta \partial_{t} \phi^{1}\right\|^{2} \\
& \leq C\left(1+\left\|\mathbf{u}^{1}\right\|_{\mathbf{H}^{k+1}}^{2}+\left\|\phi^{1}\right\|_{H^{k+1}}^{2}+\varepsilon^{2}\left\|\Delta \phi^{1}\right\|_{H^{k+1}}^{2}\right)
\end{aligned}
$$

on the time interval $\left[0, T_{\varepsilon}\right]$.

As a direct consequence of Lemma 2.1, Lemma 2.4 and 2.3, we also have:

Corollary 2.6. Let $\left(n^{1}, \mathbf{u}^{1}, \phi^{1}\right)$ be a smooth solution for the remainder system $\left(R_{\varepsilon}\right)$ and $\alpha$ an integer. There exist $\varepsilon_{1}$ and $C$ such that

$$
\left\|\partial_{t} R^{1}\right\|_{H^{k}} \leq C\left(1+\|\|\left(\mathbf{u}^{1}, \phi^{1}\right) \|_{\varepsilon, k+1}\right),
$$

for any $0<\varepsilon<\varepsilon_{1}$ and any multiindices $\alpha$ with $|\alpha|=k$.

2.3. Estimates of the $s$ order. In this subsection, we give several estimates of the $s$ order. However, the $H^{s}$-norm of the solutions depends on the $H^{s+1}$-norm and hence cannot be closed until the next subsection. The main result in this subsection is Proposition 2.10. In the following, $\gamma \geq 0$ will always denote a multiindex with $|\gamma|=s$. 
LEMMA 2.7. Let $\gamma \geq 0$ be a multiindex with $|\gamma|=s$ and $\left(n^{1}, \mathbf{u}^{1}, \phi^{1}\right)$ a smooth solution for the system (2.2). There exist $\varepsilon_{1}>0$ and $C>0$ such that

$$
\begin{gathered}
\left\{\frac{1}{2} \frac{d}{d t}\left\|\partial^{\gamma} \mathbf{u}^{1}\right\|_{L^{2}}^{2}+\frac{1}{2} \frac{d}{d t} \int \frac{n^{0}}{n^{0}+\varepsilon n^{1}}\left|\partial^{\gamma} \phi^{1}\right|^{2}+\frac{\varepsilon}{2} \frac{d}{d t} \int \frac{1}{n^{0}+\varepsilon n^{1}}\left|\partial^{\gamma} \nabla \phi^{1}\right|^{2}\right\} \\
\leq C\left(1+\left.\varepsilon^{3}\left\|\left(\mathbf{u}^{1}, \phi^{1}\right)\right\|\right|_{\varepsilon, 5} ^{3}\right)\left(1+\left\|\left|\left(\mathbf{u}^{1}, \phi^{1}\right) \|\right|_{\varepsilon, s \vee 3}^{2}\right),\right.
\end{gathered}
$$

for any $0<\varepsilon<\varepsilon_{1}$, where $s \vee 3=\max \{s, 3\}$.

Proof. Let $\gamma$ be a multiindex with $|\gamma|=s \geq 0$. Taking $\partial^{\gamma}$ to (2.2b), we obtain

$$
\partial_{t} \partial^{\gamma} \mathbf{u}^{1}+\partial^{\gamma}\left(\mathbf{u}^{0} \cdot \nabla \mathbf{u}^{1}\right)+\partial^{\gamma}\left(\mathbf{u}^{1} \cdot \nabla \mathbf{u}^{0}\right)+\varepsilon \partial^{\gamma}\left(\mathbf{u}^{1} \cdot \nabla \mathbf{u}^{1}\right)=-\partial^{\gamma} \nabla \phi^{1} .
$$

Taking the $L^{2}$ inner product with $\partial^{\gamma} \mathbf{u}^{1}$, we obtain

$$
\begin{aligned}
\int \partial_{t} \partial^{\gamma} \mathbf{u}^{1} \partial^{\gamma} \mathbf{u}^{1}= & -\int \partial^{\gamma} \nabla \phi^{1} \partial^{\gamma} \mathbf{u}^{1}-\varepsilon \int \partial^{\gamma}\left(\mathbf{u}^{1} \cdot \nabla \mathbf{u}^{1}\right) \partial^{\gamma} \mathbf{u}^{1} \\
& -\int \partial^{\gamma}\left(\mathbf{u}^{0} \cdot \nabla \mathbf{u}^{1}\right) \partial^{\gamma} \mathbf{u}^{1}-\int \partial^{\gamma}\left(\mathbf{u}^{1} \cdot \nabla \mathbf{u}^{0}\right) \partial^{\gamma} \mathbf{u}^{1} \\
= & : I+I I+I I I+I V .
\end{aligned}
$$

- Estimate of the fourth term IV.

The term $I V$ can be bounded by

$$
\begin{aligned}
I V & \leq C\left\|\partial^{\gamma}\left(\mathbf{u}^{1} \cdot \nabla \mathbf{u}^{0}\right)\right\|_{L^{2}}\left\|\partial^{\gamma} \mathbf{u}^{1}\right\|_{L^{2}} \\
& \leq C\left(\left\|\mathbf{u}^{1}\right\|_{H^{s}}\left\|\nabla \mathbf{u}^{0}\right\|_{L^{\infty}}+\left\|\nabla \mathbf{u}^{0}\right\|_{H^{s}}\left\|\mathbf{u}^{1}\right\|_{L^{\infty}}\right)\left\|\mathbf{u}^{1}\right\|_{H^{s}} \\
& \leq C\left(\left\|\mathbf{u}^{1}\right\|_{H^{s}}^{2}+\left\|\mathbf{u}^{1}\right\|_{H^{2}}^{2}\right),
\end{aligned}
$$

where we have used the commutator estimates (A.1), the Sobolev embedding $H^{2} \hookrightarrow L^{\infty}$ when $d \leq 3$ and the fact that $\left(n^{0}, \mathbf{u}^{0}\right)$ is a known smooth solution of the Euler equation (1.2) by Theorem 1.1

- Estimate of the third term III.

By integration by parts, the third term $I I I$ can be rewritten as

$$
\begin{aligned}
I I I & =-\int \mathbf{u}^{0} \cdot \nabla \partial^{\gamma} \mathbf{u}^{1} \partial^{\gamma} \mathbf{u}^{1}-\int\left[\partial^{\gamma}, \mathbf{u}^{0}\right] \cdot \nabla \mathbf{u}^{1} \partial^{\gamma} \mathbf{u}^{1} \\
& =\frac{1}{2} \int \nabla \cdot \mathbf{u}^{0}\left|\partial^{\gamma} \mathbf{u}^{1}\right|^{2}-\int\left[\partial^{\gamma}, \mathbf{u}^{0}\right] \cdot \nabla \mathbf{u}^{1} \partial^{\gamma} \mathbf{u}^{1} .
\end{aligned}
$$

By using commutator estimates (A.1), we obtain

$$
\begin{aligned}
\left|\int\left[\partial^{\gamma}, \mathbf{u}^{0}\right] \cdot \nabla \mathbf{u}^{1} \partial^{\gamma} \mathbf{u}^{1}\right| & \leq C\left\|\left[\partial^{\gamma}, \mathbf{u}^{0}\right] \cdot \nabla \mathbf{u}^{1}\right\|_{L^{2}}\left\|\partial^{\gamma} \mathbf{u}^{1}\right\|_{L^{2}} \\
& \leq C\left(\left\|\nabla \mathbf{u}^{0}\right\|_{L^{\infty}}\left\|\nabla \mathbf{u}^{1}\right\|_{H^{s-1}}+\left\|\mathbf{u}^{0}\right\|_{H^{s}}\left\|\nabla \mathbf{u}^{1}\right\|_{L^{\infty}}\right)\left\|\partial^{\gamma} \mathbf{u}^{1}\right\|_{L^{2}} \\
& \leq C\left(\left\|\mathbf{u}^{1}\right\|_{H^{3}}^{2}+\left\|\mathbf{u}^{1}\right\|_{H^{s}}^{2}\right),
\end{aligned}
$$

where we have used the Hölder inequality and the Sobolev embedding $H^{2} \hookrightarrow L^{\infty}$. This yields the estimate

$$
I I I \leq C\left(\left\|\mathbf{u}^{1}\right\|_{H^{3}}^{2}+\left\|\mathbf{u}^{1}\right\|_{H^{s}}^{2}\right),
$$

since the first term on the RHS of (2.14) is bounded by $C\left\|\mathbf{u}^{1}\right\|_{H^{s}}^{2}$. 
- Estimate of the second term II.

Similar to the estimate of $I I I$, we have by integration by parts that

$$
\begin{aligned}
I I= & \frac{\varepsilon}{2} \int \nabla \cdot \mathbf{u}^{1} \partial^{\gamma} \mathbf{u}^{1} \partial^{\gamma} \mathbf{u}^{1}-\varepsilon \int\left[\partial^{\gamma}, \mathbf{u}^{1}\right] \cdot \nabla \mathbf{u}^{1} \partial^{\gamma} \mathbf{u}^{1} \\
\leq & C \varepsilon\left\|\nabla \cdot \mathbf{u}^{1}\right\|_{L^{\infty}}\left\|\partial^{\gamma} \mathbf{u}^{1}\right\|_{L^{2}}^{2} \\
& +C \varepsilon\left(\left\|\nabla \mathbf{u}^{1}\right\|_{L^{\infty}}\left\|\nabla \mathbf{u}^{1}\right\|_{H^{s-1}}+\left\|\nabla \mathbf{u}^{1}\right\|_{L^{\infty}}\left\|\mathbf{u}^{1}\right\|_{H^{s}}\right)\left\|\partial^{\gamma} \mathbf{u}^{1}\right\|_{L^{2}} \\
\leq & C \varepsilon\left\|\mathbf{u}^{1}\right\|_{H^{3}}\left\|\mathbf{u}^{1}\right\|_{H^{s}}^{2} .
\end{aligned}
$$

- Estimate of the first term I.

By integration by parts, the term $I$ in (2.12) is rewritten as

$$
I=\int \partial^{\gamma} \phi^{1} \partial^{\gamma} \nabla \cdot \mathbf{u}^{1}
$$

To handle this term, we note that from the remainder equation (2.2a),

$$
\begin{aligned}
& \left(n^{0}+\varepsilon n^{1}\right) \partial^{\gamma} \nabla \cdot \mathbf{u}^{1}+\left[\partial^{\gamma}, n^{0}+\varepsilon n^{1}\right] \nabla \cdot \mathbf{u}^{1}+\partial_{t} \partial^{\gamma} n^{1} \\
& \quad+\partial^{\gamma}\left(\left(\mathbf{u}^{0}+\varepsilon \mathbf{u}^{1}\right) \cdot \nabla n^{1}+\mathbf{u}^{1} \cdot \nabla n^{0}\right)+\partial^{\gamma}\left(n^{1} \nabla \cdot \mathbf{u}^{0}\right)=0 .
\end{aligned}
$$

Inserting this into $I$, we obtain

$$
\begin{aligned}
I= & -\int \frac{\partial^{\gamma} \phi^{1}}{n^{0}+\varepsilon n^{1}} \partial_{t} \partial^{\gamma} n^{1}-\int \frac{\partial^{\gamma} \phi^{1}}{n^{0}+\varepsilon n^{1}} \partial^{\gamma}\left(\left(\mathbf{u}^{0}+\varepsilon \mathbf{u}^{1}\right) \cdot \nabla n^{1}\right) \\
& -\int \frac{\partial^{\gamma} \phi^{1}}{n^{0}+\varepsilon n^{1}}\left[\partial^{\gamma}, n^{0}+\varepsilon n^{1}\right] \nabla \cdot \mathbf{u}^{1}-\int \frac{\partial^{\gamma} \phi^{1}}{n^{0}+\varepsilon n^{1}} \partial^{\gamma}\left(\mathbf{u}^{1} \cdot \nabla n^{0}\right) \\
& -\int \frac{\partial^{\gamma} \phi^{1}}{n^{0}+\varepsilon n^{1}} \partial^{\gamma}\left(n^{1} \cdot \nabla \mathbf{u}^{0}\right)=: \sum_{i=1}^{5} I_{i} .
\end{aligned}
$$

In the following, we estimate $I_{3} \sim I_{5}$ while leaving the estimates of $I_{1}$ and $I_{2}$ to the next lemmas.

For $I_{3}$, we have

$$
\begin{aligned}
I_{3} & \leq C\left\|\partial^{\gamma} \phi^{1}\right\|_{L^{2}}\left(\left\|\nabla\left(n^{0}+\varepsilon n^{1}\right)\right\|_{L^{\infty}}\left\|\nabla \cdot \mathbf{u}^{1}\right\|_{H^{s-1}}+\left\|n^{0}+\varepsilon n^{1}\right\|_{H^{s}}\left\|\nabla \cdot \mathbf{u}^{1}\right\|_{L^{\infty}}\right) \\
& \leq C\left\|\phi^{1}\right\|_{H^{s}}\left(\left\|\mathbf{u}^{1}\right\|_{H^{s}}+\left\|\mathbf{u}^{1}\right\|_{H^{3}}\right)+C \varepsilon\left(\left\|n^{1}\right\|_{H^{3}}+\left\|\mathbf{u}^{1}\right\|_{H^{3}}\right)\left\|\phi^{1}\right\|_{H^{s}}\left(\left\|n^{1}\right\|_{H^{s}}+\left\|\mathbf{u}^{1}\right\|_{H^{s}}\right) \\
& \leq C\left(1+\varepsilon\left\|\left(n^{1}, \mathbf{u}^{1}\right)\right\|_{H^{3}}\right)\left\|\left(n^{1}, \mathbf{u}^{1}, \phi^{1}\right)\right\|_{H^{s}}^{2}+C\left\|\mathbf{u}^{1}\right\|_{H^{3}}^{2},
\end{aligned}
$$

where we have used the Hölder inequality, commutator estimates (A.1) and the fact that $n^{0}$ and $n^{0}+\varepsilon n^{1}$ are bounded from above and below by positive numbers when $\varepsilon<\varepsilon_{1}$ is small enough in (2.5).

For $I_{4}$, directly applying the Hölder inequality and Lemma A.1 yields

$$
\begin{aligned}
I_{24} & \leq C\left\|\phi^{1}\right\|_{H^{s}}\left(\left\|\mathbf{u}^{1}\right\|_{L^{\infty}}\left\|\nabla n^{0}\right\|_{H^{s}}+\left\|\mathbf{u}^{1}\right\|_{H^{s}}\left\|\nabla n^{0}\right\|_{L^{\infty}}\right) \\
& \leq C\left\|\phi^{1}\right\|_{H^{s}}^{2}+C\left\|\mathbf{u}^{1}\right\|_{H^{s}}^{2}+C\left\|\mathbf{u}^{1}\right\|_{H^{2}}^{2} .
\end{aligned}
$$

Similarly, $I_{5}$ can be bounded by

$$
I_{25} \leq C\left\|\phi^{1}\right\|_{H^{s}}^{2}+C\left\|n^{1}\right\|_{H^{s}}^{2}+C\left\|n^{1}\right\|_{H^{2}}^{2} .
$$


Summarizing, we have that

$$
I \leq I_{1}+I_{2}+C\left\|\left(n^{1}, \mathbf{u}^{1}\right)\right\|_{H^{3}}^{2}+C\left(1+\varepsilon^{2}\left\|\left(n^{1}, \mathbf{u}^{1}\right)\right\|_{H^{3}}^{2}\right)\left\|\left(n^{1}, \mathbf{u}^{1}, \phi^{1}\right)\right\|_{H^{s}}^{2} .
$$

To end the proof of Lemma 2.7 we need to get suitable estimates for $I_{1}$ and $I_{2}$. However, this is not straightforward, and to make it easier to read, we leave the proof to the next two lemmas.

Lemma 2.8. The term of $I_{2}$ in (2.18) is bounded by

$$
I_{21} \leq C\left(1+\varepsilon^{3}\left|\left\|\left(\mathbf{u}^{1}, \phi^{1}\right) \mid\right\|_{\varepsilon, 4}^{3}\right)\left(1+\|\|\left(\mathbf{u}^{1}, \phi^{1}\right) \mid \|_{\varepsilon, s \vee 3}^{2}\right),\right.
$$

for some constant $C>0$ and for all $0<\varepsilon<\varepsilon_{1}$.

Proof. First, we observe that $I_{2}$ in (2.18) can be decomposed into

$$
I_{2}=-\int \frac{\partial^{\gamma} \phi^{1}\left(\mathbf{u}^{0}+\varepsilon \mathbf{u}^{1}\right)}{n^{0}+\varepsilon n^{1}} \cdot \nabla \partial^{\gamma} n^{1}-\int \frac{\partial^{\gamma} \phi^{1}}{n^{0}+\varepsilon n^{1}}\left[\partial^{\gamma}, \mathbf{u}^{0}+\varepsilon \mathbf{u}^{1}\right] \cdot \nabla n^{1}=: I_{21}+I_{22} .
$$

By commutator estimate (A.1),

$$
\begin{aligned}
I_{22} & \leq C\left\|\partial^{\gamma} \phi^{1}\right\|\left(\left\|\nabla \mathbf{u}^{0}+\varepsilon \nabla \mathbf{u}^{1}\right\|_{L^{\infty}}\left\|\nabla n^{1}\right\|_{H^{s-1}}+\left\|\mathbf{u}^{0}+\varepsilon \mathbf{u}^{1}\right\|_{H^{s}}\left\|\nabla n^{1}\right\|_{L^{\infty}}\right) \\
& \leq C\left\|\phi^{1}\right\|_{H^{s}}\left(\left\|n^{1}\right\|_{H^{s}}+\left\|n^{1}\right\|_{H^{3}}\right)+C \varepsilon\left(\left\|\left(n^{1}, \mathbf{u}^{1}\right)\right\|_{H^{3}}\right)\left\|\phi^{1}\right\|_{H^{s}}\left(\left\|\left(n^{1}, \mathbf{u}^{1}\right)\right\|_{H^{s}}\right) \\
& \leq C\left\|n^{1}\right\|_{H^{3}}^{2}+C\left(1+\varepsilon\left\|\left(n^{1}, \mathbf{u}^{1}\right)\right\|_{H^{3}}\right)\left\|\left(n^{1}, \mathbf{u}^{1}, \phi^{1}\right)\right\|_{H^{s}}^{2} .
\end{aligned}
$$

To treat $I_{21}$, we first note that from the remainder equation (2.2c),

$$
\partial^{\gamma} \nabla n^{1}=\nabla \partial^{\gamma}\left(n^{0} \phi^{1}\right)-\varepsilon \partial^{\gamma} \nabla \Delta \phi^{1}-\partial^{\gamma} \nabla \Delta \phi^{0}-\sqrt{\varepsilon} \partial^{\gamma} \nabla R^{1} .
$$

Hence $I_{21}$ is accordingly divided into

$$
\begin{aligned}
I_{21}= & -\int \frac{\partial^{\gamma} \phi^{1}\left(\mathbf{u}^{0}+\varepsilon \mathbf{u}^{1}\right)}{n^{0}+\varepsilon n^{1}} \cdot \nabla \partial^{\gamma}\left(n^{0} \phi^{1}\right)+\varepsilon \int \frac{\partial^{\gamma} \phi^{1}\left(\mathbf{u}^{0}+\varepsilon \mathbf{u}^{1}\right)}{n^{0}+\varepsilon n^{1}} \cdot \partial^{\gamma} \nabla \Delta \phi^{1} \\
& +\int \frac{\partial^{\gamma} \phi^{1}\left(\mathbf{u}^{0}+\varepsilon \mathbf{u}^{1}\right)}{n^{0}+\varepsilon n^{1}} \cdot \partial^{\gamma} \nabla \Delta \phi^{0}+\sqrt{\varepsilon} \int \frac{\partial^{\gamma} \phi^{1}\left(\mathbf{u}^{0}+\varepsilon \mathbf{u}^{1}\right)}{n^{0}+\varepsilon n^{1}} \cdot \partial^{\gamma} \nabla R^{1} \\
= & : \sum_{i=1}^{4} I_{21 i} .
\end{aligned}
$$

For the first term $I_{211}$, we have

$$
\begin{aligned}
I_{211} & =-\int \frac{\partial^{\gamma} \phi^{1}\left(\mathbf{u}^{0}+\varepsilon \mathbf{u}^{1}\right)}{n^{0}+\varepsilon n^{1}} \cdot n^{0} \nabla \partial^{\gamma} \phi^{1}-\int \frac{\partial^{\gamma} \phi^{1}\left(\mathbf{u}^{0}+\varepsilon \mathbf{u}^{1}\right)}{n^{0}+\varepsilon n^{1}} \cdot\left[\partial^{\gamma}, n^{0}\right] \nabla \phi^{1} \\
& =\frac{1}{2} \int \nabla \cdot\left(\frac{n^{0}\left(\mathbf{u}^{0}+\varepsilon \mathbf{u}^{1}\right)}{n^{0}+\varepsilon n^{1}}\right)\left|\partial^{\gamma} \phi^{1}\right|^{2}-\int \frac{\partial^{\gamma} \phi^{1}\left(\mathbf{u}^{0}+\varepsilon \mathbf{u}^{1}\right)}{n^{0}+\varepsilon n^{1}} \cdot\left[\partial^{\gamma}, n^{0}\right] \nabla \phi^{1} .
\end{aligned}
$$

By direct computation and Sobolev embedding, we have

$$
\left\|\nabla \cdot\left(\frac{n^{0}\left(\mathbf{u}^{0}+\varepsilon \mathbf{u}^{1}\right)}{n^{0}+\varepsilon n^{1}}\right)\right\|_{L^{\infty}} \leq C+C \varepsilon^{2}\left(\left\|n^{1}\right\|_{H^{3}}^{2}+\left\|\mathbf{u}^{1}\right\|_{H^{3}}^{2}\right),
$$


which yields

$$
\begin{aligned}
I_{211} \leq & C\left\|\phi^{1}\right\|_{H^{s}}^{2}+C \varepsilon^{2}\left(\left\|n^{1}\right\|_{H^{3}}^{2}+\left\|\mathbf{u}^{1}\right\|_{H^{3}}^{2}\right)\left\|\phi^{1}\right\|_{H^{s}}^{2}+C\left\|\partial^{\gamma} \phi^{1}\right\|_{L^{2}}^{2} \\
& \cdot\left(1+\varepsilon\left\|\mathbf{u}^{1}\right\|_{L^{\infty}}\right)\left(\left\|\nabla n^{0}\right\|_{L^{\infty}}\left\|\nabla \phi^{1}\right\|_{H^{s-1}}+\left\|n^{0}\right\|_{H^{s}}\left\|\nabla \phi^{1}\right\|_{L^{\infty}}\right) \\
\leq & C\left(1+\varepsilon^{2}\left(\left\|n^{1}\right\|_{H^{3}}^{2}+\left\|\mathbf{u}^{1}\right\|_{H^{3}}^{2}\right)\right)\left\|\phi^{1}\right\|_{H^{s}}^{2}+C\left\|\phi^{1}\right\|_{H^{3}}^{2},
\end{aligned}
$$

thanks to the commutator estimates (A.1). For $I_{212}$, by integration by parts, we obtain

$$
\begin{aligned}
I_{212} & =\varepsilon \int \frac{\left(\mathbf{u}^{0}+\varepsilon \mathbf{u}^{1}\right) \partial^{\gamma} \phi^{1}}{n^{0}+\varepsilon n^{1}} \cdot \partial^{\gamma} \nabla \Delta \phi^{1} \\
& =-\varepsilon \int \frac{\left(\mathbf{u}^{0}+\varepsilon \mathbf{u}^{1}\right)}{n^{0}+\varepsilon n^{1}} \cdot \partial^{\gamma} \nabla \phi^{1} \cdot \partial^{\gamma} \nabla \nabla \phi^{1}-\varepsilon \int \nabla\left(\frac{\left(\mathbf{u}^{0}+\varepsilon \mathbf{u}^{1}\right)}{n^{0}+\varepsilon n^{1}}\right) \partial^{\gamma} \phi^{1} \cdot \partial^{\gamma} \nabla \nabla \phi^{1} \\
& =\frac{3 \varepsilon}{2} \int \nabla \cdot\left(\frac{\left(\mathbf{u}^{0}+\varepsilon \mathbf{u}^{1}\right)}{n^{0}+\varepsilon n^{1}}\right)\left|\partial^{\gamma} \nabla \phi^{1}\right|^{2}+\varepsilon \int \nabla^{2}\left(\frac{\left(\mathbf{u}^{0}+\varepsilon \mathbf{u}^{1}\right)}{n^{0}+\varepsilon n^{1}}\right) \partial^{\gamma} \phi^{1} \partial^{\gamma} \nabla \phi^{1} .
\end{aligned}
$$

By direct computation and the Sobolev embedding $H^{2} \hookrightarrow L^{\infty}$, we have

$$
\begin{gathered}
\left\|\nabla \cdot\left(\frac{\left(\mathbf{u}^{0}+\varepsilon \mathbf{u}^{1}\right)}{n^{0}+\varepsilon n^{1}}\right)\right\|_{L^{\infty}} \leq C+C \varepsilon^{2}\left(\left\|n^{1}\right\|_{H^{3}}^{2}+\left\|\mathbf{u}^{1}\right\|_{H^{3}}^{2}\right), \\
\left\|\nabla^{2}\left(\frac{\left(\mathbf{u}^{0}+\varepsilon \mathbf{u}^{1}\right)}{n^{0}+\varepsilon n^{1}}\right)\right\|_{L^{\infty}} \leq C+C \varepsilon^{3}\left(\left\|n^{1}\right\|_{H^{4}}^{3}+\left\|\mathbf{u}^{1}\right\|_{H^{4}}^{3}\right),
\end{gathered}
$$

which yield

$$
I_{212} \leq C\left(1+\varepsilon^{3}\left(\left\|\left(n^{1}, \mathbf{u}^{1}\right)\right\|_{H^{4}}^{3}\right)\right)\left(\left\|\phi^{1}\right\|_{H^{s}}^{2}+\varepsilon\left\|\nabla \phi^{1}\right\|_{H^{s}}^{2}\right) .
$$

For $I_{213}$, by Hölder inequality, we obtain

$$
I_{213} \leq C\left(1+\left\|\mathbf{u}^{1}\right\|_{L^{2}}^{2}+\left\|\phi^{1}\right\|_{H^{s}}^{2}\right) .
$$

For $I_{214}$, by integration by parts,

$$
I_{214}=-\sqrt{\varepsilon} \int \nabla \cdot\left(\frac{\left(\mathbf{u}^{0}+\varepsilon \mathbf{u}^{1}\right)}{n^{0}+\varepsilon n^{1}}\right) \partial^{\gamma} \phi^{1} \partial^{\gamma} R^{1}-\sqrt{\varepsilon} \int \frac{\left(\mathbf{u}^{0}+\varepsilon \mathbf{u}^{1}\right)}{n^{0}+\varepsilon n^{1}} \cdot \nabla \partial^{\gamma} \phi^{1} \partial^{\gamma} R^{1} .
$$

By using Lemma 2.1, we obtain

$$
\begin{aligned}
I_{214} \leq & C\left(1+\varepsilon^{2}\left\|\left(n^{1}, \mathbf{u}^{1}\right)\right\|_{H^{3}}^{2}\right)\left\|\phi^{1}\right\|_{H^{s}}^{2} \\
& +C\left(1+\varepsilon\left\|\mathbf{u}^{1}\right\|_{L^{\infty}}\right)\left(\left\|\phi^{1}\right\|_{H^{s}}^{2}+\varepsilon\left\|\nabla \phi^{1}\right\|_{H^{s}}^{2}\right) \\
\leq & C\left(1+\varepsilon\left\|\left(\mathbf{u}^{1}, n^{1}\right)\right\|_{H^{3}}\right)\left(\left\|\phi^{1}\right\|_{H^{s}}^{2}+\varepsilon\left\|\nabla \phi^{1}\right\|_{H^{s}}^{2}\right) .
\end{aligned}
$$

By (2.23) and putting (2.24)-(2.27) together, we obtain

$$
\begin{aligned}
I_{21} \leq & C+C\left\|\left(n^{1}, \mathbf{u}^{1}, \phi^{1}\right)\right\|_{H^{3}}^{2} \\
& +C\left(1+\varepsilon^{3}\left\|\left(n^{1}, \mathbf{u}^{1}\right)\right\|_{H^{4}}^{3}\right)\left(\left\|\left(n^{1}, \mathbf{u}^{1}, \phi^{1}\right)\right\|_{H^{s}}^{2}+\varepsilon\left\|\nabla \phi^{1}\right\|_{H^{s}}^{2}\right) \\
\leq & C\left(1+\varepsilon^{3}\|\|\left(\mathbf{u}^{1}, \phi^{1}\right) \|_{\varepsilon, 4}^{3}\right)\left(1+\|\|\left(\mathbf{u}^{1}, \phi^{1}\right) \|_{\varepsilon, s \vee 3}^{2}\right),
\end{aligned}
$$

where we have used the definition of the norm $\||\cdot|\|_{\varepsilon, s}$ in (1.7) and Lemma 2.2 to replace the norms of $n^{1}$ with the norms of $\phi^{1}$. By putting (2.21) and (2.28) together and using Lemma 2.2. we obtain (2.20). This ends the proof of Lemma 2.8. 
Lemma 2.9. For $I_{1}$ in (2.18), we have the estimates

$$
\begin{aligned}
I_{1} \leq & -\frac{1}{2} \frac{d}{d t} \int \frac{n^{0}}{n^{\varepsilon}}\left|\partial^{\gamma} \phi^{1}\right|^{2}-\frac{\varepsilon}{2} \frac{d}{d t} \int \frac{1}{n^{\varepsilon}}\left|\partial^{\gamma} \nabla \phi^{1}\right|^{2} \\
& +C\left(1+\left.\varepsilon^{3}||\left|\left(\mathbf{u}^{1}, \phi^{1}\right)\right|\right|_{\varepsilon, 5} ^{3}\right)\left(1+\left.||\left|\left(\mathbf{u}^{1}, \phi^{1}\right)\right|\right|_{\varepsilon, s \vee 3} ^{2}\right) .
\end{aligned}
$$

Proof. From the remainder equation (2.2c), we obtain

$$
\partial^{\gamma} \partial_{t} n^{1}=\partial^{\gamma} \partial_{t}\left(n^{0} \phi^{1}\right)-\varepsilon \partial^{\gamma} \partial_{t} \Delta \phi^{1}-\partial^{\gamma} \partial_{t} \Delta \phi^{0}-\sqrt{\varepsilon} \partial^{\gamma} \partial_{t} R^{1}
$$

In this way, we can divide $I_{1}$ in (2.18) into the following:

$$
\begin{aligned}
I_{1}= & \varepsilon \int \frac{\partial^{\gamma} \phi^{1}}{n^{0}+\varepsilon n^{1}} \partial^{\gamma} \partial_{t} \Delta \phi^{1}-\int \frac{\partial^{\gamma} \phi^{1}}{n^{0}+\varepsilon n^{1}} \partial^{\gamma} \partial_{t}\left(n^{0} \phi^{1}\right) \\
& -\int \frac{\partial^{\gamma} \phi^{1}}{n^{0}+\varepsilon n^{1}} \partial^{\gamma} \partial_{t} \Delta \phi^{0}-\sqrt{\varepsilon} \int \frac{\partial^{\gamma} \phi^{1}}{n^{0}+\varepsilon n^{1}} \partial^{\gamma} \partial_{t} R^{1}=\sum_{i=1}^{4} I_{1 i} .
\end{aligned}
$$

In the following, we treat the RHS terms of (2.30) one by one.

- Estimate of $I_{12}$.

For the term $I_{12}$, we have

$$
\begin{aligned}
I_{12} & =-\int \frac{\partial^{\gamma} \phi^{1}}{n^{0}+\varepsilon n^{1}} \partial^{\gamma}\left(\phi^{1} \partial_{t} n^{0}\right)-\int \frac{\partial^{\gamma} \phi^{1}}{n^{0}+\varepsilon n^{1}} \partial^{\gamma}\left(n^{0} \partial_{t} \phi^{1}\right) \\
& =-\int \frac{\partial^{\gamma} \phi^{1}}{n^{0}+\varepsilon n^{1}} \partial^{\gamma}\left(\phi^{1} \partial_{t} n^{0}\right)-\int \frac{n^{0} \partial^{\gamma} \phi^{1}}{n^{0}+\varepsilon n^{1}} \partial_{t} \partial^{\gamma} \phi^{1}-\int \frac{\partial^{\gamma} \phi^{1}}{n^{0}+\varepsilon n^{1}}\left[\partial^{\gamma}, n^{0}\right] \partial_{t} \phi^{1} \\
& =: \sum_{i=1}^{3} I_{12 i} .
\end{aligned}
$$

For the first term $I_{121}$, since $n^{0}$ is known and is assumed to be smooth in Theorem 1.1. we have

$$
I_{121} \leq C\left\|\phi^{1}\right\|_{H^{s}}^{2}
$$

thanks to the multiplicative estimate in Lemma A.1 For the second term $I_{122}$, by integration by parts and Lemma 2.3, we have

$$
\begin{aligned}
I_{122} & =-\frac{1}{2} \frac{d}{d t} \int \frac{n^{0}}{n^{0}+\varepsilon n^{1}}\left|\partial^{\gamma} \phi^{1}\right|^{2}+\frac{1}{2} \int \partial_{t}\left(\frac{n^{0}}{n^{0}+\varepsilon n^{1}}\right)\left|\partial^{\gamma} \phi^{1}\right|^{2} \\
& \leq-\frac{1}{2} \frac{d}{d t} \int \frac{n^{0}}{n^{0}+\varepsilon n^{1}}\left|\partial^{\gamma} \phi^{1}\right|^{2}+C\left(1+\varepsilon\left\|\partial_{t} n^{1}\right\|_{L^{\infty}}\right)\left\|\partial^{\gamma} \phi^{1}\right\|_{L^{2}}^{2} \\
& \leq-\frac{1}{2} \frac{d}{d t} \int \frac{n^{0}}{n^{0}+\varepsilon n^{1}}\left|\partial^{\gamma} \phi^{1}\right|^{2}+C\left(1+\left.\varepsilon^{2}\left\|||\left(\mathbf{u}^{1}, \phi^{1}\right)\right\|\right|_{\varepsilon, 3} ^{2}\right)\left\|\phi^{1}\right\|_{H^{s}}^{2}
\end{aligned}
$$

where we have used the Sobolev embedding $H^{2} \hookrightarrow L^{\infty}$ for $d \leq 3$. For the third term $I_{123}$, by commutator estimates in Lemma A.1 and Lemma 2.3, we have

$$
\begin{aligned}
I_{123} & \leq C\left\|\partial^{\gamma} \phi^{1}\right\|_{L^{2}}\left(\left\|\nabla n^{0}\right\|_{L^{\infty}}\left\|\partial_{t} \phi^{1}\right\|_{H^{s-1}}+\left\|n^{0}\right\|_{H^{s}}\left\|\partial_{t} \phi^{1}\right\|_{L^{\infty}}\right) \\
& \leq C\left(1+\left\|\left(\mathbf{u}^{1}, \phi^{1}\right)\right\|\left\|_{\varepsilon, s}^{2}+\right\|\left(\left\|\left(\mathbf{u}^{1}, \phi^{1}\right)\right\| \|_{\varepsilon, 3}^{2}\right) .\right.
\end{aligned}
$$


Summarizing, we have

$$
\begin{aligned}
I_{12} \leq & -\frac{1}{2} \frac{d}{d t} \int \frac{n^{0}}{n^{0}+\varepsilon n^{1}}\left|\partial^{\gamma} \phi^{1}\right|^{2} \\
& +C\left(1+\varepsilon^{2}\left|\left\|\left(\mathbf{u}^{1}, \phi^{1}\right) \mid\right\|_{\varepsilon, 3}^{2}\right)\left(1+\left|\left\|\left(\mathbf{u}^{1}, \phi^{1}\right)\left|\left\|_{\varepsilon, s}^{2}+\right\|\right|\left|\left(\mathbf{u}^{1}, \phi^{1}\right)\right|\right\|_{\varepsilon, 3}^{2}\right) .\right.\right.
\end{aligned}
$$

- Estimate of $I_{13}$.

For the term $I_{13}$, since $\phi^{0}$ is known and smooth, it is easy to obtain

$$
I_{13} \leq C\left(1+\left\|\phi^{1}\right\|_{H^{s}}^{2}\right) .
$$

- Estimate of $I_{14}$.

For the term $I_{14}$, by integration by parts, we have

$$
I_{14}=\sqrt{\varepsilon} \int \frac{\partial^{\gamma+\gamma_{1}} \phi^{1}}{n^{0}+\varepsilon n^{1}} \partial^{\gamma-\gamma_{1}} \partial_{t} R^{1}+\sqrt{\varepsilon} \int \partial^{\gamma_{1}}\left(\frac{1}{n^{0}+\varepsilon n^{1}}\right) \partial^{\gamma} \phi^{1} \partial^{\gamma-\gamma_{1}} \partial_{t} R^{1},
$$

where $\gamma_{1} \leq \gamma$ is a multiindex with $\left|\gamma_{1}\right|=1$. By Lemma 2.2. Corollary 2.6 and the definition of the triple norm ||$|\cdot|||_{\varepsilon, s}$, we have the bound

$$
\begin{aligned}
I_{14} & \leq C \sqrt{\varepsilon}\left\|\partial^{\gamma+\gamma_{1}} \phi^{1}\right\|_{L^{2}}\left\|\partial_{t} R^{1}\right\|_{H^{s-1}}+C\left(1+\varepsilon\left\|\partial^{\gamma_{1}} n^{1}\right\|_{L^{\infty}}\right)\left\|\partial^{\gamma} \phi^{1}\right\|_{L^{2}}\left\|\partial_{t} R^{1}\right\|_{H^{s-1}} \\
& \leq C\left\|\partial_{t} R^{1}\right\|_{H^{s-1}}^{2}+C \varepsilon\left\|\nabla \phi^{1}\right\|_{H^{s}}^{2}+C\left(1+\varepsilon^{2}\left\|n^{1}\right\|_{H^{3}}^{2}\right)\left\|\phi^{1}\right\|_{H^{s}}^{2} \\
& \leq C\left(1+\varepsilon^{2}\|\|\left(\mathbf{u}^{1}, \phi^{1}\right)\|\|_{\varepsilon, 3}^{2}\right)\left(1+\left\|\left(\mathbf{u}^{1}, \phi^{1}\right)\right\| \|_{\varepsilon, s}^{2}\right) .
\end{aligned}
$$

- Estimate of $I_{11}$.

We next deal with the term $I_{11}$ in (2.30). By integration by parts, we have

$$
\begin{aligned}
I_{11}= & -\varepsilon \int \frac{1}{n^{0}+\varepsilon n^{1}} \partial^{\gamma} \nabla \phi^{1} \cdot \partial_{t} \partial^{\gamma} \nabla \phi^{1}-\varepsilon \int \nabla\left(\frac{1}{n^{0}+\varepsilon n^{1}}\right) \partial^{\gamma} \phi^{1} \cdot \partial_{t} \partial^{\gamma} \nabla \phi^{1} \\
= & -\frac{\varepsilon}{2} \frac{d}{d t} \int \frac{1}{n^{0}+\varepsilon n^{1}}\left|\partial^{\gamma} \nabla \phi^{1}\right|^{2}+\frac{\varepsilon}{2} \int \partial_{t}\left(\frac{1}{n^{0}+\varepsilon n^{1}}\right)\left|\partial^{\gamma} \nabla \phi^{1}\right|^{2} \\
& -\varepsilon \int \nabla\left(\frac{1}{n^{0}+\varepsilon n^{1}}\right) \partial^{\gamma} \phi^{1} \cdot \partial_{t} \partial^{\gamma} \nabla \phi^{1}=: I_{111}+I_{112}+I_{113} .
\end{aligned}
$$

Since from Sobolev embedding and Lemma 2.3, we have

$$
\left\|\partial_{t}\left(\frac{n^{0}}{n^{0}+\varepsilon n^{1}}\right)\right\|_{L^{\infty}} \leq C\left(1+\varepsilon\left\|\partial_{t} n^{1}\right\|_{L^{\infty}}\right) \leq C\left(1+\varepsilon^{2}\left\|\mid\left(\mathbf{u}^{1}, \phi^{1}\right)\right\| \|_{\varepsilon, 3}^{2}\right),
$$

it is immediate that

$$
I_{112} \leq C\left(1+\varepsilon^{2}\left\|\left(\mathbf{u}^{1}, \phi^{1}\right)\right\| \|_{\varepsilon, 3}^{2}\right)\left(\varepsilon\left\|\partial^{\gamma} \nabla \phi^{1}\right\|^{2}\right) .
$$

For the term $I_{113}$, we have by integration by parts,

$$
\begin{aligned}
I_{113}= & \varepsilon \int \Delta\left(\frac{1}{n^{0}+\varepsilon n^{1}}\right) \partial^{\gamma} \phi^{1} \partial_{t} \partial^{\gamma} \phi^{1}+\varepsilon \int \nabla\left(\frac{1}{n^{0}+\varepsilon n^{1}}\right) \partial^{\gamma} \nabla \phi^{1} \partial_{t} \partial^{\gamma} \phi^{1} \\
= & -\varepsilon \int \partial^{\gamma_{1}} \Delta\left(\frac{1}{n^{\varepsilon}}\right) \partial^{\gamma} \phi^{1} \partial_{t} \partial^{\gamma-\gamma_{1}} \phi^{1}-\varepsilon \int \Delta\left(\frac{1}{n^{\varepsilon}}\right) \partial^{\gamma+\gamma_{1}} \phi^{1} \partial_{t} \partial^{\gamma-\gamma_{1}} \phi^{1} \\
& -\varepsilon \int \partial^{\gamma_{1}} \nabla\left(\frac{1}{n^{\varepsilon}}\right) \nabla \partial^{\gamma} \phi^{1} \partial_{t} \partial^{\gamma-\gamma_{1}} \phi^{1}-\varepsilon \int \nabla\left(\frac{1}{n^{\varepsilon}}\right) \nabla \partial^{\gamma+\gamma_{1}} \phi^{1} \partial_{t} \partial^{\gamma-\gamma_{1}} \phi^{1},
\end{aligned}
$$


where $\gamma_{1} \leq \gamma$ is a multiindex with $\left|\gamma_{1}\right|=1$. By direct computation, Hölder inequality and Sobolev embedding $H^{2} \hookrightarrow L^{\infty}$, it is easy to obtain

$$
\begin{aligned}
\left\|\partial^{\alpha}\left(\frac{1}{n^{\varepsilon}}\right)\right\|_{L^{\infty}} & \leq C\left(1+\varepsilon^{|\alpha|}\left\|n^{1}\right\|_{H^{2+|\alpha|}}^{|\alpha|}\right) \\
& \leq C\left(1+\varepsilon^{|\alpha|}\left\|||\left(\mathbf{u}^{1}, \phi^{1}\right) \mid\right\|_{\varepsilon, 2+|\alpha|}^{|\alpha|}\right)
\end{aligned}
$$

for any smooth function $n^{0}$ and any multiindex $\alpha$, thanks to Lemma 2.2. On the other hand, from Lemma 2.4 and Lemma 2.3, we obtain

$$
\begin{aligned}
\left\|\partial_{t} \partial^{\gamma-\gamma_{1}} \phi^{1}\right\|_{L^{2}}^{2} & \leq C\left(1+\left\|\phi^{1}\right\|_{H^{s-1}}^{2}+\left\|\partial_{t} n^{1}\right\|_{H^{s-1}}^{2}\right) \\
& \leq C\left(1+\left\|\left(\mathbf{u}^{1}, \phi^{1}\right)\right\| \|_{\varepsilon, s}^{2}\right) .
\end{aligned}
$$

Since the order of the derivatives on $n^{0} /\left(n^{0}+\varepsilon n^{1}\right)$ in (2.34) does not exceed 3, by using Hölder inequality, (2.35) and (2.36), $I_{113}$ can be bounded by

$$
\begin{aligned}
I_{113} \leq & C\left(1+\varepsilon^{3}\left\|\left|\left(\mathbf{u}^{1}, \phi^{1}\right) \|\right|_{\varepsilon, 5}^{3}\right) \cdot \varepsilon\left(\left\|\partial^{\gamma} \phi^{1}\right\|_{L^{2}}+\left\|\partial^{\gamma+\gamma_{1}} \phi^{1}\right\|_{L^{2}}\right.\right. \\
& \left.+\left\|\nabla \partial^{\gamma} \phi^{1}\right\|_{L^{2}}+\left\|\nabla \partial^{\gamma+\gamma_{1}} \phi^{1}\right\|_{L^{2}}\right) \cdot\left\|\partial_{t} \partial^{\gamma-\gamma_{1}} \phi^{1}\right\|_{L^{2}} \\
\leq & C\left(1+\varepsilon^{3}\left\|\left|\left(\mathbf{u}^{1}, \phi^{1}\right) \|\right|_{\varepsilon, 5}^{3}\right)\right. \\
& \times\left(\left\|\partial^{\gamma} \phi^{1}\right\|_{L^{2}}^{2}+\varepsilon\left\|\partial^{\gamma} \nabla \phi^{1}\right\|_{L^{2}}^{2}+\varepsilon^{2}\left\|\partial^{\gamma} \Delta \phi^{1}\right\|_{L^{2}}^{2}+\left\|\partial_{t} \partial^{\gamma-\gamma_{1}} \phi^{1}\right\|_{L^{2}}^{2}\right) \\
\leq & C\left(1+\varepsilon^{3}\left\|\left|\left(\mathbf{u}^{1}, \phi^{1}\right) \|\right|_{\varepsilon, 5}^{3}\right)\left(1+\left\|\left(\mathbf{u}^{1}, \phi^{1}\right)\right\|_{\varepsilon, s}^{2}\right),\right.
\end{aligned}
$$

where we have used the definition of $\||\cdot|\|_{\varepsilon, s}$ in (1.7) and the $L^{2}$ boundedness of the Riesz operator 23. To be more precise, there exists some constant $C>0$ such that $\left\|\partial_{i} \partial_{j} \phi^{1}\right\| \leq C\left\|\Delta \phi^{1}\right\|$ since $\partial_{i} \partial_{j}=-R_{i} R_{j} \Delta$, where $R_{i}$ is the $i^{\text {th }}$ Riesz operator. In particular, we have $\left\|\nabla \partial^{\gamma+\gamma_{1}} \phi^{1}\right\| \leq C\left\|\partial^{\gamma} \Delta \phi\right\|$.

Summarizing, we have

$$
I_{11}=-\frac{\varepsilon}{2} \frac{d}{d t} \int \frac{1}{n^{\varepsilon}}\left|\partial^{\gamma} \nabla \phi^{1}\right|^{2}+C\left(1+\varepsilon^{3} \|\left.\left|\left(\mathbf{u}^{1}, \phi^{1}\right)\right|\right|_{\varepsilon, 5} ^{3}\right)\left(1+\|\left.\left|\left(\mathbf{u}^{1}, \phi^{1}\right)\right|\right|_{\varepsilon, s} ^{2}\right) .
$$

By (2.30) and the estimates of (2.31), (2.32), (2.33) and (2.37), we have

$$
\begin{aligned}
I_{1} \leq & -\frac{1}{2} \frac{d}{d t} \int \frac{n^{0}}{n^{\varepsilon}}\left|\partial^{\gamma} \phi^{1}\right|^{2}-\frac{\varepsilon}{2} \frac{d}{d t} \int \frac{1}{n^{\varepsilon}}\left|\partial^{\gamma} \nabla \phi^{1}\right|^{2} \\
& +C\left(1+\varepsilon^{3} \|\left.\left|\left(\mathbf{u}^{1}, \phi^{1}\right)\right|\right|_{\varepsilon, 5} ^{3}\right)\left(1+\left\|\mid\left(\mathbf{u}^{1}, \phi^{1}\right)\right\|_{\varepsilon, s \vee 3}^{2}\right) .
\end{aligned}
$$

This ends the proof of Lemma 2.9

Now, we can end the proof of Lemma 2.7.

End of proof of Lemma 2.7. The proof of Lemma 2.7 is closed by (2.12), (2.13), (2.15), (2.16), (2.19) and Lemma 2.8 and 2.9.

Proposition 2.10. Let $s \geq 0$ be a positive integer and $\left(n^{1}, \mathbf{u}^{1}, \phi^{1}\right)$ a smooth solution for the system (2.2). There exists $\varepsilon_{1}>0$ and $C, C^{\prime}>0$ such that for any $0<\varepsilon<\varepsilon_{1}$, there holds

$$
\begin{aligned}
\left\|\mathbf{u}^{1}(t)\right\|_{H^{s}}^{2} & +\left\|\phi^{1}(t)\right\|_{H^{s}}^{2}+\varepsilon\left\|\nabla \phi^{1}(t)\right\|_{H^{s}}^{2} \\
\leq & C^{\prime}\left(\left\|\mathbf{u}^{1}(0)\right\|_{H^{s}}^{2}+\left\|\phi^{1}(0)\right\|_{H^{s}}^{2}+\varepsilon\left\|\nabla \phi^{1}(0)\right\|_{H^{s}}^{2}\right) \\
& \quad+C \int_{0}^{t}\left(1+\varepsilon^{3}\left\|\left(\mathbf{u}^{1}, \phi^{1}\right)\right\| \|_{\varepsilon, 5}^{3}\right)\left(1+\|\|\left(\mathbf{u}^{1}, \phi^{1}\right)\|\|_{\varepsilon, s \vee 3}^{2}\right) d \tau
\end{aligned}
$$


where $C^{\prime}$ depends only on $\sigma^{\prime}$ and $\sigma^{\prime \prime}$.

Proof. This is shown by integrating (2.11) over $[0, t]$ and summing them up for $|\gamma| \leq s$, and then using $\sigma^{\prime}<n^{0}<\sigma^{\prime \prime}$ and $\frac{\sigma^{\prime}}{2}<n^{\varepsilon}<2 \sigma^{\prime \prime}$ for any $t \in\left[0, T_{\varepsilon}\right]$ in (1.4) and (2.5) for $0<\varepsilon<\varepsilon_{1}$.

However, this Gronwall inequality is not closed since the right hand side of (2.38) depends on $\varepsilon^{2}\left\|\Delta \phi^{1}\right\|_{H^{s}}^{2}$, which does not appear on the left hand side. This will be treated in the next subsection.

2.4. Weighted $s+1$ order estimates. In the following, we also let $\gamma$ be a multiindex with $|\gamma|=s$. The main result in this subsection is Proposition 2.14.

Lemma 2.11. Let $s \geq 0$ be a positive integer and $\left(n^{1}, \mathbf{u}^{1}, \phi^{1}\right)$ a smooth solution for the system (2.2). There exists $\varepsilon_{1}>0$ and $C, C^{\prime}>0$ such that

$$
\begin{aligned}
\frac{\varepsilon}{2} \frac{d}{d t} \int\left|\partial^{\gamma} \nabla \mathbf{u}^{1}\right|^{2} \leq & -\frac{\varepsilon}{2} \frac{d}{d t} \int \frac{n^{0}}{n^{\varepsilon}}\left|\partial^{\gamma} \nabla \phi^{1}\right|^{2}-\frac{\varepsilon^{2}}{2} \frac{d}{d t} \int \frac{1}{n^{\varepsilon}}\left|\partial^{\gamma} \Delta \phi^{1}\right|^{2} \\
& +C\left(1+\left.\varepsilon^{2}||\left|\left(\mathbf{u}^{1}, \phi^{1}\right)\right|\right|_{\varepsilon, 4} ^{2}\right)\left(1+\|\left.\left|\left(\mathbf{u}^{1}, \phi^{1}\right)\right|\right|_{\varepsilon, s \vee 3} ^{2}\right),
\end{aligned}
$$

for any $0<\varepsilon<\varepsilon_{1}$.

Proof. Let $\gamma$ be a multiindex with $|\gamma|=s \geq 0$. Taking $\partial^{\gamma}$ in the second equation of (2.2), we obtain

$$
\partial_{t} \partial^{\gamma} \mathbf{u}^{1}+\partial^{\gamma}\left(\mathbf{u}^{0} \cdot \nabla \mathbf{u}^{1}\right)+\partial^{\gamma}\left(\mathbf{u}^{1} \cdot \nabla \mathbf{u}^{0}\right)+\varepsilon \partial^{\gamma}\left(\mathbf{u}^{1} \cdot \nabla \mathbf{u}^{1}\right)=-\partial^{\gamma} \nabla \phi^{1} .
$$

Taking the $L^{2}$ inner product with $-\varepsilon \partial^{\gamma} \Delta \mathbf{u}^{1}$, we obtain

$$
\begin{aligned}
\frac{\varepsilon}{2} \frac{d}{d t} \int\left|\partial^{\gamma} \nabla \mathbf{u}^{1}\right|^{2}= & -\varepsilon \int \partial_{t} \partial^{\gamma} \mathbf{u}^{1} \partial^{\gamma} \Delta \mathbf{u}^{1} \\
= & \varepsilon \int \partial^{\gamma} \nabla \phi^{1} \partial^{\gamma} \Delta \mathbf{u}^{1}+\varepsilon^{2} \int \partial^{\gamma}\left(\mathbf{u}^{1} \cdot \nabla \mathbf{u}^{1}\right) \partial^{\gamma} \Delta \mathbf{u}^{1} \\
& +\varepsilon \int \partial^{\gamma}\left(\mathbf{u}^{0} \cdot \nabla \mathbf{u}^{1}\right) \partial^{\gamma} \Delta \mathbf{u}^{1}+\varepsilon \int \partial^{\gamma}\left(\mathbf{u}^{1} \cdot \nabla \mathbf{u}^{0}\right) \partial^{\gamma} \Delta \mathbf{u}^{1} \\
= & : I^{\varepsilon}+I I^{\varepsilon}+I I I^{\varepsilon}+I V^{\varepsilon}
\end{aligned}
$$

- Estimate of $I V^{\varepsilon}$.

The term $I V^{\varepsilon}$ can be bounded by

$$
\begin{aligned}
I V^{\varepsilon} & =-\varepsilon \int \partial^{\gamma} \nabla\left(\mathbf{u}^{1} \cdot \nabla \mathbf{u}^{0}\right) \partial^{\gamma} \nabla \mathbf{u}^{1} \\
& =-\varepsilon \int\left(\partial^{\gamma}\left(\nabla \mathbf{u}^{1} \cdot \nabla \mathbf{u}^{0}\right)+\partial^{\gamma}\left(\mathbf{u}^{1} \cdot \nabla^{2} \mathbf{u}^{0}\right)\right) \partial^{\gamma} \nabla \mathbf{u}^{1} \\
& \leq C \varepsilon\left(\left\|\partial^{\gamma}\left(\nabla \mathbf{u}^{1} \cdot \nabla \mathbf{u}^{0}\right)\right\|_{L^{2}}^{2}+\left\|\partial^{\gamma}\left(\mathbf{u}^{1} \cdot \nabla^{2} \mathbf{u}^{0}\right)\right\|_{L^{2}}^{2}\right)+C \varepsilon\left\|\partial^{\gamma} \nabla \mathbf{u}^{1}\right\|_{L^{2}}^{2} \\
& \leq C\left(\left\|\mathbf{u}^{1}\right\|_{H^{s}}^{2}+\varepsilon\left\|\nabla \mathbf{u}^{1}\right\|_{H^{s}}^{2}+\left\|\mathbf{u}^{1}\right\|_{H^{3}}^{2}\right),
\end{aligned}
$$

where we have used the commutator estimates (A.1), the Sobolev embedding $H^{2} \hookrightarrow L^{\infty}$ when $d \leq 3$ and the fact that $n^{0}$ and $\mathbf{u}^{0}$ are known smooth solutions of the Euler equation (1.2) by Theorem 1.1 
- Estimate of $I I I^{\varepsilon}$.

By integration by parts, the third term $I I I^{\varepsilon}$ can be rewritten as

$$
\begin{aligned}
I I I^{\varepsilon}= & \varepsilon \int \mathbf{u}^{0} \cdot \nabla \partial^{\gamma} \mathbf{u}^{1} \partial^{\gamma} \Delta \mathbf{u}^{1}+\varepsilon \int\left[\partial^{\gamma}, \mathbf{u}^{0}\right] \cdot \nabla \mathbf{u}^{1} \partial^{\gamma} \Delta \mathbf{u}^{1} \\
= & \varepsilon \int \sum_{i, j, k} u_{i}^{0} \partial_{i} \partial^{\gamma} u_{k}^{1} \partial^{\gamma} \partial_{j} \partial_{j} u_{k}^{1}+\varepsilon \int \sum_{\beta=1}^{\gamma} C_{\gamma}^{\beta} \partial^{\beta} \mathbf{u}^{0} \cdot \partial^{\gamma-\beta} \nabla \mathbf{u}^{1} \cdot \partial^{\gamma} \Delta \mathbf{u}^{1} \\
= & -2 \varepsilon \int \sum_{i, j, k} \partial_{j} u_{i}^{0} \partial_{i} \partial^{\gamma} u_{k}^{1} \partial^{\gamma} \partial_{j} u_{k}^{1}+\varepsilon \int \sum_{i, j, k} \partial_{i} u_{i}^{0} \partial^{\gamma} \partial_{j} u_{k}^{1} \partial^{\gamma} \partial_{j} u_{k}^{1} \\
& -\varepsilon \int \sum_{\beta=1}^{\gamma} C_{\gamma}^{\beta} \partial^{\beta} \mathbf{u}^{0} \cdot \partial^{\gamma-\beta} \nabla^{2} \mathbf{u}^{1} \cdot \partial^{\gamma} \nabla \mathbf{u}^{1}-\varepsilon \int \sum_{\beta=1}^{\gamma} C_{\gamma}^{\beta} \partial^{\beta} \nabla \mathbf{u}^{0} \cdot \partial^{\gamma-\beta} \nabla \mathbf{u}^{1} \cdot \partial^{\gamma} \nabla \mathbf{u}^{1} \\
= & : I I I_{1}^{\varepsilon}+I I I_{2}^{\varepsilon}+I I I_{3}^{\varepsilon}+I I I_{4}^{\varepsilon},
\end{aligned}
$$

where $\mathbf{u}^{0}=\left(u_{1}^{0}, \cdots, u_{d}^{0}\right)$ and $\mathbf{u}^{1}=\left(u_{1}^{1}, \cdots, u_{d}^{1}\right)$ and we have used integration by parts twice in the third equality. For $I I I_{1}^{\varepsilon}, I I I_{2}^{\varepsilon}$ and $I I I_{3}^{\varepsilon}$, we easily obtain

$$
\left|I I I_{1}^{\varepsilon}, I I I_{2}^{\varepsilon}, I I I_{3}^{\varepsilon}\right| \leq C \varepsilon\left\|\nabla \mathbf{u}^{1}\right\|_{H^{s}}^{2} .
$$

For $I I I_{4}^{\varepsilon}$, by Hölder inequality, we obtain

$$
\left|I I I_{4}^{\varepsilon}\right| \leq C \varepsilon\left\|\mathbf{u}^{1}\right\|_{H^{s}}\left\|\nabla \mathbf{u}^{1}\right\|_{H^{s}} \leq C\left(\left\|\mathbf{u}^{1}\right\|_{H^{s}}^{2}+\varepsilon\left\|\nabla \mathbf{u}^{1}\right\|_{H^{s}}^{2}\right) .
$$

Summing them up, we obtain

$$
\left|I I I^{\varepsilon}\right| \leq C\left(\left\|\mathbf{u}^{1}\right\|_{H^{s}}^{2}+\varepsilon\left\|\nabla \mathbf{u}^{1}\right\|_{H^{s}}^{2}\right) .
$$

- Estimate of $I I^{\varepsilon}$.

For the second term $I I^{\varepsilon}$, by integration by parts, we have

$$
\begin{aligned}
I I^{\varepsilon}= & \varepsilon^{2} \int \mathbf{u}^{1} \cdot \partial^{\gamma} \nabla \mathbf{u}^{1} \partial^{\gamma} \Delta \mathbf{u}^{1}+\varepsilon^{2} \int\left[\partial^{\gamma}, \mathbf{u}^{1}\right] \cdot \nabla \mathbf{u}^{1} \partial^{\gamma} \Delta \mathbf{u}^{1} \\
= & -\varepsilon^{2} \int \sum_{i, j, k} \partial_{j} u_{i}^{1} \partial^{\gamma} \partial_{i} u_{k}^{1} \partial^{\gamma} \partial_{j} u_{k}^{1}+\frac{\varepsilon^{2}}{2} \int \sum_{i, j, k} \partial_{i} u_{i}^{1} \partial^{\gamma} \partial_{j} u_{k}^{1} \partial^{\gamma} \partial_{j} u_{k}^{1} \\
& -\varepsilon^{2} \int\left[\partial^{\gamma}, \nabla \mathbf{u}^{1}\right] \cdot \nabla \mathbf{u}^{1} \cdot \partial^{\gamma} \nabla \mathbf{u}^{1}-\varepsilon^{2} \int\left[\partial^{\gamma}, \mathbf{u}^{1}\right] \cdot \nabla^{2} \mathbf{u}^{1} \cdot \partial^{\gamma} \nabla \mathbf{u}^{1}=: \sum_{i=1}^{4} I I_{i}^{\varepsilon} .
\end{aligned}
$$

By Hölder inequality, $I I_{1}^{\varepsilon}$ and $I I_{2}^{\varepsilon}$ are bounded by

$$
\begin{aligned}
\left|I I_{1}^{\varepsilon}, I I_{2}^{\varepsilon}\right| & \leq C\left(\varepsilon\left\|\nabla \mathbf{u}^{1}\right\|_{L^{\infty}}\right)\left(\varepsilon\left\|\nabla \mathbf{u}^{1}\right\|_{H^{s}}^{2}\right) \\
& \leq C\left(\varepsilon\left\|\mathbf{u}^{1}\right\|_{H^{3}}\right)\left(\varepsilon\left\|\nabla \mathbf{u}^{1}\right\|_{H^{s}}^{2}\right) .
\end{aligned}
$$

By commutator estimates,

$$
\begin{aligned}
\left\|\left[\partial^{\gamma}, \nabla \mathbf{u}^{1}\right] \cdot \nabla \mathbf{u}^{1}\right\|_{L^{2}} & \leq C\left(\left\|\nabla^{2} \mathbf{u}^{1}\right\|_{L^{\infty}}\left\|\nabla \mathbf{u}^{1}\right\|_{H^{s-1}}+\left\|\nabla \mathbf{u}^{1}\right\|_{H^{s}}\left\|\nabla \mathbf{u}^{1}\right\|_{L^{\infty}}\right) \\
& \leq C\left\|\mathbf{u}^{1}\right\|_{H^{4}}\left(\left\|\mathbf{u}^{1}\right\|_{H^{s}}+\left\|\nabla \mathbf{u}^{1}\right\|_{H^{s}}\right) .
\end{aligned}
$$

Hence

$$
\left|I I_{3}^{\varepsilon}\right| \leq C \varepsilon\left\|\mathbf{u}^{1}\right\|_{H^{4}}\left(\left\|\mathbf{u}^{1}\right\|_{H^{s}}^{2}+\varepsilon\left\|\nabla \mathbf{u}^{1}\right\|_{H^{s}}^{2}\right) .
$$


Similarly, by commutator estimates

$$
\begin{aligned}
\left|I I_{4}^{\varepsilon}\right| & \leq C \varepsilon^{2}\left(\left\|\nabla \mathbf{u}^{1}\right\|_{L^{\infty}}\left\|\nabla^{2} \mathbf{u}^{1}\right\|_{H^{s-1}}+\left\|\mathbf{u}^{1}\right\|_{H^{s}}\left\|\nabla^{2} \mathbf{u}^{1}\right\|_{L^{\infty}}\right)\left\|\nabla \mathbf{u}^{1}\right\|_{H^{s}} \\
& \leq C \varepsilon\left\|\mathbf{u}^{1}\right\|_{H^{4}}\left(\left\|\mathbf{u}^{1}\right\|_{H^{s}}^{2}+\varepsilon\left\|\nabla \mathbf{u}^{1}\right\|_{H^{s}}^{2}\right) .
\end{aligned}
$$

Summing them up, we obtain

$$
\left|I I^{\varepsilon}\right| \leq C \varepsilon\left\|\mathbf{u}^{1}\right\|_{H^{4}}\left(\left\|\mathbf{u}^{1}\right\|_{H^{s}}^{2}+\varepsilon\left\|\nabla \mathbf{u}^{1}\right\|_{H^{s}}^{2}\right) .
$$

- Estimate of $I^{\varepsilon}$.

In the following, we treat the first term $I^{\varepsilon}$ in (2.40). By integration by parts thrice, we obtain

$$
I^{\varepsilon}=-\varepsilon \int \partial^{\gamma} \Delta \phi^{1} \partial^{\gamma} \nabla \cdot \mathbf{u}^{1}
$$

By using (2.17), we obtain

$$
\begin{aligned}
I^{\varepsilon}= & \int \frac{\varepsilon \partial^{\gamma} \Delta \phi^{1}}{n^{\varepsilon}} \partial_{t} \partial^{\gamma} n^{1}+\int \frac{\varepsilon \partial^{\gamma} \Delta \phi^{1}}{n^{\varepsilon}} \partial^{\gamma}\left(\mathbf{u}^{\varepsilon} \cdot \nabla n^{1}\right) \\
& +\int \frac{\varepsilon \partial^{\gamma} \Delta \phi^{1}}{n^{\varepsilon}}\left[\partial^{\gamma}, n^{\varepsilon}\right] \nabla \cdot \mathbf{u}^{1}+\int \frac{\varepsilon \partial^{\gamma} \Delta \phi^{1}}{n^{\varepsilon}} \partial^{\gamma}\left(\mathbf{u}^{1} \cdot \nabla n^{0}\right) \\
& +\int \frac{\varepsilon \partial^{\gamma} \Delta \phi^{1}}{n^{\varepsilon}} \partial^{\gamma}\left(n^{1} \cdot \nabla \mathbf{u}^{0}\right)=: \sum_{i=1}^{5} I_{i}^{\varepsilon} .
\end{aligned}
$$

In the following, we estimate $I_{3}^{\varepsilon} \sim I_{5}^{\varepsilon}$ while leaving the estimates of $I_{1}^{\varepsilon}$ and $I_{2}^{\varepsilon}$ to the next two lemmas. For $I_{3}^{\varepsilon}$, we have

$$
\begin{aligned}
I_{3}^{\varepsilon} & \leq C \varepsilon\left\|\partial^{\gamma} \Delta \phi^{1}\right\|_{L^{2}}\left(\left\|\nabla\left(n^{0}+\varepsilon n^{1}\right)\right\|_{L^{\infty}}\left\|\nabla \cdot \mathbf{u}^{1}\right\|_{H^{s-1}}+\left\|n^{0}+\varepsilon n^{1}\right\|_{H^{s}}\left\|\nabla \cdot \mathbf{u}^{1}\right\|_{L^{\infty}}\right) \\
& \leq C \varepsilon^{2}\left\|\Delta \phi^{1}\right\|_{H^{s}}^{2}+\left\|\mathbf{u}^{1}\right\|_{H^{s}}^{2}+\left\|\mathbf{u}^{1}\right\|_{H^{3}}^{2}+C \varepsilon^{2}\left(\left\|n^{1}\right\|_{H^{3}}^{2}+\left\|\mathbf{u}^{1}\right\|_{H^{3}}^{2}\left(\left\|n^{1}\right\|_{H^{s}}^{2}+\left\|\mathbf{u}^{1}\right\|_{H^{s}}^{2}\right),\right.
\end{aligned}
$$

where we have used the commutator estimates (A.1) and the fact that $n^{0}+\varepsilon n^{1}$ are bounded from above and below by positive numbers when $\varepsilon<\varepsilon_{1}$ is small enough in (2.5). Recalling Lemma 2.2 and the definition of the triple norm (1.7), we obtain

$$
I_{3}^{\varepsilon} \leq C\left(1+\varepsilon^{2}\left\|\left|\left(\mathbf{u}^{1}, \phi\right)\right|\right\|_{\varepsilon, 3}^{2}\right)\left|\left\|( \mathbf { u } ^ { 1 } , \phi ) \left|\left\|\left.\right|_{\varepsilon, s} ^{2}+C\right\| \mathbf{u}^{1} \|_{H^{3}}^{2} .\right.\right.\right.
$$

For $I_{4}^{\varepsilon}$, we have

$$
\begin{aligned}
I_{4}^{\varepsilon} & \leq C \varepsilon\left\|\Delta \phi^{1}\right\|_{H^{s}}\left(\left\|\mathbf{u}^{1}\right\|_{L^{\infty}}\left\|\nabla n^{0}\right\|_{H^{s}}+\left\|\mathbf{u}^{1}\right\|_{H^{s}}\left\|\nabla n^{0}\right\|_{L^{\infty}}\right) \\
& \leq C \varepsilon^{2}\left\|\Delta \phi^{1}\right\|_{H^{s}}^{2}+C\left\|\mathbf{u}^{1}\right\|_{H^{s}}^{2}+C\left\|\mathbf{u}^{1}\right\|_{H^{2}}^{2} .
\end{aligned}
$$

Similarly, $I_{5}^{\varepsilon}$ can be bounded by

$$
\begin{aligned}
I_{5}^{\varepsilon} & \leq C \varepsilon\left\|\Delta \phi^{1}\right\|_{H^{s}}^{2}+C\left\|n^{1}\right\|_{H^{s}}^{2}+C\left\|n^{1}\right\|_{H^{2}}^{2} \\
& \leq C\left(1+\|\phi\| \|_{\varepsilon, s}^{2}\right)+C\left\|n^{1}\right\|_{H^{2}}^{2},
\end{aligned}
$$

thanks to Lemma 2.2. Summarizing, we obtain from (2.44)

$$
\begin{aligned}
I^{\varepsilon} \leq & I_{1}^{\varepsilon}+I_{2}^{\varepsilon}+C\left\|\mathbf{u}^{1}\right\|_{H^{3}}^{2}+C\left\|n^{1}\right\|_{H^{2}}^{2} \\
& +C\left(1+\varepsilon^{2}\|\|\left(\mathbf{u}^{1}, \phi\right)\|\|_{\varepsilon, 3}^{2}\right)\left(1+\left\|\mid\left(\mathbf{u}^{1}, \phi\right)\right\| \|_{\varepsilon, s}^{2}\right),
\end{aligned}
$$

where $I_{1}^{\varepsilon}$ and $I_{2}^{\varepsilon}$ will be treated in the next two lemmas. 
Lemma 2.12. The term $I_{2}^{\varepsilon}$ in (2.44) can be estimated as

$$
I_{2}^{\varepsilon} \leq C\left(1+\left.\varepsilon^{2}||\left|\left(\mathbf{u}^{1}, \phi^{1}\right)\right|\right|_{\varepsilon, 3} ^{2}\right)\left(1+\|\left.\left|\left(\mathbf{u}^{1}, \phi^{1}\right)\right|\right|_{\varepsilon, s} ^{2}\right)+\||| \phi^{1}||_{\varepsilon, 3}^{2},
$$

for all $0<\varepsilon<\varepsilon_{1}$ for some $\varepsilon_{1}>0$.

Proof. Recall that $I_{2}^{\varepsilon}$ is given by (2.44). By integration by parts, it can be rewritten as

$$
I_{2}^{\varepsilon}=\int \frac{\varepsilon \partial^{\gamma} \Delta \phi^{1}}{n^{\varepsilon}} \mathbf{u}^{\varepsilon} \cdot \nabla \partial^{\gamma} n^{1}+\int \frac{\varepsilon \partial^{\gamma} \Delta \phi^{1}}{n^{\varepsilon}}\left[\partial^{\gamma}, \mathbf{u}^{\varepsilon}\right] \cdot \nabla n^{1}=: I_{21}^{\varepsilon}+I_{22}^{\varepsilon} .
$$

- Estimate of $I_{22}^{\varepsilon}$.

For the second term $I_{22}^{\varepsilon}$, we obtain

$$
\begin{aligned}
I_{22}^{\varepsilon} & \leq C \varepsilon\left\|\partial^{\gamma} \Delta \phi^{1}\right\|_{L^{2}}\left\|\left[\partial^{\gamma}, \mathbf{u}^{\varepsilon}\right] \cdot \nabla n^{1}\right\|_{L^{2}} \\
& \leq C \varepsilon\left\|\partial^{\gamma} \Delta \phi^{1}\right\|_{L^{2}}\left(\left\|\nabla \mathbf{u}^{\varepsilon}\right\|_{L^{\infty}}\left\|\nabla n^{1}\right\|_{H^{s-1}}+\left\|\mathbf{u}^{\varepsilon}\right\|_{H^{s}}\left\|\nabla n^{1}\right\|_{L^{\infty}}\right) \\
& \leq C \varepsilon\left\|\Delta \phi^{1}\right\|_{H^{s}}\left(\left\|n^{1}\right\|_{H^{s}}+\left\|n^{1}\right\|_{H^{3}}+\varepsilon\left\|\mathbf{u}^{1}\right\|_{H^{3}}\left\|n^{1}\right\|_{H^{s}}+\varepsilon\left\|n^{1}\right\|_{H^{3}}\left\|\mathbf{u}^{1}\right\|_{H^{s}}\right) \\
& \leq C \varepsilon^{2}\left\|\Delta \phi^{1}\right\|_{H^{s}}^{2}+C\left(\left\|n^{1}\right\|_{H^{s}}^{2}+\left\|n^{1}\right\|_{H^{3}}^{2}+\varepsilon^{2}\left\|\left(\mathbf{u}^{1}, n^{1}\right)\right\|_{H^{3}}^{2}\left(\left\|\left(n^{1}, \mathbf{u}^{1}\right)\right\|_{H^{s}}^{2}\right)\right) \\
& \leq C\left(1+\varepsilon^{2}\left\|\mid\left(\mathbf{u}^{1}, \phi^{1}\right)\right\|_{\varepsilon, 3}^{2}\right)\left(1+\left\|\left(\mathbf{u}^{1}, \phi^{1}\right)\right\| \|_{\varepsilon, s}^{2}\right)+\left\|\phi^{1}\right\|_{\varepsilon, 3}^{2},
\end{aligned}
$$

thanks to the commutator estimates (A.1) in the second step, Lemma2.2 in the last step and the definition of the triple norm in (1.7).

- Estimate of $I_{21}^{\varepsilon}$.

In the rest of this lemma, we focus on the treatment of $I_{21}^{\varepsilon}$. Recalling the remainder equation (2.2c) (see also (2.22) $), I_{21}^{\varepsilon}$ can be divided into

$$
\begin{aligned}
I_{21}^{\varepsilon}= & \int \frac{\varepsilon \partial^{\gamma} \Delta \phi^{1}}{n^{\varepsilon}} \mathbf{u}^{\varepsilon} \cdot \nabla \partial^{\gamma}\left(n^{0} \phi^{1}\right)-\varepsilon^{2} \int \frac{\partial^{\gamma} \Delta \phi^{1}}{n^{\varepsilon}} \mathbf{u}^{\varepsilon} \cdot \partial^{\gamma} \nabla \Delta \phi^{1} \\
& -\int \frac{\varepsilon \partial^{\gamma} \Delta \phi^{1}}{n^{\varepsilon}} \mathbf{u}^{\varepsilon} \cdot \partial^{\gamma} \nabla \Delta \phi^{0}-\sqrt{\varepsilon} \int \frac{\varepsilon \partial^{\gamma} \Delta \phi^{1}}{n^{\varepsilon}} \mathbf{u}^{\varepsilon} \cdot \partial^{\gamma} \nabla R^{1}=: \sum_{i=1}^{4} I_{21 i}^{\varepsilon} .
\end{aligned}
$$

In the following, we estimate the four terms on the RHS of (2.47). For the first term $I_{211}^{\varepsilon}$, we have

$$
\begin{aligned}
I_{211}^{\varepsilon}= & \int \frac{\varepsilon n^{0} \partial^{\gamma} \Delta \phi^{1}}{n^{\varepsilon}} \mathbf{u}^{\varepsilon} \cdot \nabla \partial^{\gamma} \phi^{1}+\int \frac{\varepsilon \partial^{\gamma} \Delta \phi^{1}}{n^{\varepsilon}} \mathbf{u}^{\varepsilon} \cdot\left[\partial^{\gamma}, n^{0}\right] \nabla \phi^{1} \\
= & \frac{\varepsilon}{2} \int \nabla \cdot\left(\frac{n^{0} \mathbf{u}^{\varepsilon}}{n^{\varepsilon}}\right)\left|\partial^{\gamma} \nabla \phi^{1}\right|^{2}-\varepsilon \int \sum_{i, j} \partial_{i}\left(\frac{n^{0} u_{j}^{\varepsilon}}{n^{\varepsilon}}\right) \partial^{\gamma} \partial_{i} \phi^{1} \partial^{\gamma} \partial_{j} \phi^{1} \\
& +\int \frac{\varepsilon \partial^{\gamma} \Delta \phi^{1}}{n^{\varepsilon}} \mathbf{u}^{\varepsilon} \cdot\left[\partial^{\gamma}, n^{0}\right] \nabla \phi^{1}=: I_{2111}^{\varepsilon}+I_{2112}^{\varepsilon}+I_{2113}^{\varepsilon},
\end{aligned}
$$

where $u_{j}^{\varepsilon}=\left(\mathbf{u}^{\varepsilon}\right)_{j}=u_{j}^{0}+\varepsilon u_{j}^{1}$. By using Sobolev embedding $H^{2} \hookrightarrow L^{\infty}$,

$$
\left\|\nabla\left(\frac{n^{0} \mathbf{u}^{\varepsilon}}{n^{\varepsilon}}\right)\right\|_{L^{\infty}} \leq C+C \varepsilon^{2}\left(\left\|n^{1}\right\|_{H^{3}}^{2}+\left\|\mathbf{u}^{1}\right\|_{H^{3}}^{2}\right)
$$


which yields for the first two terms on the RHS of (2.48)

$$
\begin{aligned}
\left|I_{2111}^{\varepsilon}, I_{2112}^{\varepsilon}\right| & \leq C \varepsilon\left(1+\varepsilon^{2}\left(\left\|n^{1}\right\|_{H^{3}}^{2}+\left\|\mathbf{u}^{1}\right\|_{H^{3}}^{2}\right)\right)\left\|\nabla \phi^{1}\right\|_{H^{s}}^{2} \\
& \leq C\left(1+\varepsilon^{2}\|\|\left(\mathbf{u}^{1}, \phi^{1}\right) \mid \|_{\varepsilon, 3}^{2}\right)\left|\left\|\phi^{1} \mid\right\|_{\varepsilon, s}^{2} .\right.
\end{aligned}
$$

For the term $I_{2113}^{\varepsilon}$, by the commutator estimates (A.1), we have

$$
\begin{aligned}
\left\|\left[\partial^{\gamma}, n^{0}\right] \nabla \phi^{1}\right\|_{L^{2}} & \leq C\left(\left\|\nabla n^{0}\right\|_{L^{\infty}}\left\|\nabla \phi^{1}\right\|_{H^{s-1}}+\left\|n^{0}\right\|_{H^{s}}\left\|\nabla \phi^{1}\right\|_{L^{\infty}}\right) \\
& \leq C\left(\left\|\phi^{1}\right\|_{H^{3}}+\left\|\phi^{1}\right\|_{H^{s}}\right),
\end{aligned}
$$

which implies that by Hölder inequality

$$
\begin{aligned}
I_{2113}^{\varepsilon} & \leq C\left\|\frac{1}{n^{0}+\varepsilon n^{1}}\right\|_{L^{\infty}}\left\|\varepsilon \partial^{\gamma} \Delta \phi^{1}\left(\mathbf{u}^{0}+\varepsilon \mathbf{u}^{1}\right)\right\|_{L^{2}}\left\|\left[\partial^{\gamma}, n^{0}\right] \nabla \phi^{1}\right\|_{L^{2}} \\
& \leq C\left(\left\|\phi^{1}\right\|_{H^{3}}^{2}+\left\|\phi^{1}\right\|_{H^{s}}^{2}\right)+C\left(1+\varepsilon^{2}\left\|\mathbf{u}^{1}\right\|_{L^{\infty}}^{2}\right)\left(\varepsilon^{2}\left\|\partial^{\gamma} \Delta \phi^{1}\right\|_{L^{2}}^{2}\right) \\
& \leq C\left(1+\varepsilon^{2}\left\|\mathbf{u}^{1}\right\|_{H^{2}}^{2}\right)\left\|\phi^{1}\right\|_{\varepsilon, s}^{2}+C\left\|\phi^{1}\right\|_{H^{3}}^{2},
\end{aligned}
$$

thanks to (2.5), the Sobolev embedding $H^{2} \hookrightarrow L^{\infty}$ and the definition of the triple norm (1.7).

For the first term $I_{212}$, by integration by parts and Lemma 2.2, we have

$$
\begin{aligned}
\left|I_{212}^{\varepsilon}\right| & =\left.\left|\frac{\varepsilon^{2}}{2} \int \nabla \cdot\left(\frac{\mathbf{u}^{0}+\varepsilon \mathbf{u}^{1}}{n^{0}+\varepsilon n^{1}}\right)\right| \partial^{\gamma} \Delta \phi^{1}\right|^{2} \mid \\
& \leq C\left(1+\varepsilon^{2}\left\|\left(n^{1}, \mathbf{u}^{1}\right)\right\|_{H^{3}}^{2}\right) \cdot \varepsilon^{2}\left\|\partial^{\gamma} \Delta \phi^{1}\right\|_{L^{2}}^{2} \\
& \leq C\left(1+\varepsilon^{2}\left\|\left|\left(\mathbf{u}^{1}, \phi^{1}\right) \|\right|_{\varepsilon, 3}^{2}\right)\left|\left\|\phi^{1}\right\|\right|_{\varepsilon, s}^{2} .\right.
\end{aligned}
$$

For the third term $I_{213}^{\varepsilon}$, by Hölder inequality and Sobolev embedding, we have

$$
\begin{aligned}
\left|I_{213}^{\varepsilon}\right| & \leq\left\|\frac{\varepsilon \partial^{\gamma} \Delta \phi^{1}\left(\mathbf{u}^{0}+\varepsilon \mathbf{u}^{1}\right)}{n^{0}+\varepsilon n^{1}}\right\|_{L^{2}}\left\|\partial^{\gamma} \nabla \Delta \phi^{0}\right\|_{L^{2}} \\
& \leq\left\|\partial^{\gamma} \nabla \Delta \phi^{0}\right\|_{L^{2}}^{2}+C\left(1+\varepsilon^{2}\left\|\mathbf{u}^{1}\right\|_{L^{\infty}}^{2}\right)\left(\varepsilon^{2}\left\|\partial^{\gamma} \Delta \phi^{1}\right\|_{L^{2}}^{2}\right) \\
& \leq C+C\left(1+\varepsilon^{2}\left\|\mathbf{u}^{1}\right\|_{H^{2}}^{2}\right)\left\|\phi^{1}\right\| \|_{\varepsilon, s}^{2} .
\end{aligned}
$$

For the fourth term $I_{214}^{\varepsilon}$, we have by Hölder inequality,

$$
\begin{aligned}
I_{214}^{\varepsilon} & \leq C\left\|\sqrt{\varepsilon} \partial^{\gamma} \nabla R^{1}\right\|_{L^{2}}^{2}+C\left(1+\varepsilon^{2}\left\|\mathbf{u}^{1}\right\|_{L^{\infty}}^{2}\right)\left(\varepsilon^{2}\left\|\partial^{\gamma} \Delta \phi^{1}\right\|_{H^{s}}^{2}\right) \\
& \leq C \varepsilon\left(\left\|\nabla \phi^{1}\right\|_{H^{s}}^{2}+\left\|\phi^{1}\right\|^{2}\right)+C\left(1+\varepsilon^{2}\left\|\mathbf{u}^{1}\right\|_{H^{2}}^{2}\right)\left(\varepsilon^{2}\left\|\partial^{\gamma} \Delta \phi^{1}\right\|_{L^{2}}^{2}\right) \\
& \leq C\left(1+\varepsilon^{2}\left\|\mathbf{u}^{1}\right\|_{H^{2}}^{2}\right)\left\|\phi^{1}\right\| \|_{\varepsilon, s}^{2},
\end{aligned}
$$

where we have used Lemma 2.1 with $|\alpha|=s+1$ there and the fact that $\left\|\phi^{1}\right\|_{H^{s+1}} \approx$ $\left\|\nabla \phi^{1}\right\|_{H^{s}}+\left\|\phi^{1}\right\|_{H^{s}}$. Summarizing, we obtain

$$
I_{21}^{\varepsilon} \leq C+C\left\|\phi^{1}\right\|_{H^{3}}^{2}+C\left(1+\varepsilon^{2}\|\|\left(\mathbf{u}^{1}, \phi^{1}\right) \mid \|_{\varepsilon, 3}^{2}\right)\left|\left\|\phi^{1} \mid\right\|_{\varepsilon, s}^{2} .\right.
$$

Putting (2.46) and (2.49) together, we end the proof of Lemma 2.12 ,

Lemma 2.13. The term $I_{1}^{\varepsilon}$ in (2.44) is bounded by

$$
\begin{aligned}
I_{1}^{\varepsilon} \leq & -\frac{\varepsilon}{2} \frac{d}{d t} \int\left(\frac{n^{0}}{n^{\varepsilon}}\right)\left|\partial^{\gamma} \nabla \phi^{1}\right|^{2}-\frac{\varepsilon^{2}}{2} \frac{d}{d t} \int \frac{1}{n^{\varepsilon}}\left|\partial^{\gamma} \Delta \phi^{1}\right|^{2} \\
& +C\left(1+\left.\varepsilon^{2}||\left|\left(\mathbf{u}^{1}, \phi^{1}\right)\right|\right|_{\varepsilon, 4} ^{2}\right)\left(1+\|\left.\left|\left(\mathbf{u}^{1}, \phi^{1}\right)\right|\right|_{\varepsilon, s \vee 3} ^{2}\right),
\end{aligned}
$$


for all $0<\varepsilon<\varepsilon_{1}$, for some $\varepsilon_{1}>0$.

Proof. Recall that $I_{1}^{\varepsilon}$ is given in (2.44). From the remainder equation (2.2c) (see also $(2.29)$ ), $I_{1}^{\varepsilon}$ can be divided into the following:

$$
\begin{aligned}
I_{1}^{\varepsilon}= & -\varepsilon \int \frac{\varepsilon \partial^{\gamma} \Delta \phi^{1}}{n^{0}+\varepsilon n^{1}} \partial^{\gamma} \partial_{t} \Delta \phi^{1}+\int \frac{\varepsilon \partial^{\gamma} \Delta \phi^{1}}{n^{0}+\varepsilon n^{1}} \partial^{\gamma} \partial_{t}\left(n^{0} \phi^{1}\right) \\
& +\int \frac{\varepsilon \partial^{\gamma} \Delta \phi^{1}}{n^{0}+\varepsilon n^{1}} \partial^{\gamma} \partial_{t} \Delta \phi^{0}+\sqrt{\varepsilon} \int \frac{\varepsilon \partial^{\gamma} \Delta \phi^{1}}{n^{0}+\varepsilon n^{1}} \partial^{\gamma} \partial_{t} R^{1}=: \sum_{i=1}^{4} I_{1 i}^{\varepsilon} .
\end{aligned}
$$

In the following, we will estimate the RHS terms one by one.

- Estimate of $I_{11}^{\varepsilon}$.

For the first term $I_{11}^{\varepsilon}$, we have

$$
I_{11}^{\varepsilon}=-\frac{\varepsilon^{2}}{2} \frac{d}{d t} \int \frac{1}{n^{0}+\varepsilon n^{1}}\left|\partial^{\gamma} \Delta \phi^{1}\right|^{2}+\frac{\varepsilon^{2}}{2} \int \partial_{t}\left(\frac{1}{n^{0}+\varepsilon n^{1}}\right)\left|\partial^{\gamma} \Delta \phi^{1}\right|^{2}
$$

Using Lemma 2.3 and Sobolev embedding, we have

$$
\left\|\partial_{t}\left(\frac{1}{n^{0}+\varepsilon n^{1}}\right)\right\|_{L^{\infty}} \leq C\left(1+\varepsilon\left\|\partial_{t} n^{1}\right\|_{L^{\infty}}\right) \leq C\left(1+\varepsilon^{2}\left\|\mid\left(\mathbf{u}^{1}, \phi^{1}\right)\right\| \|_{\varepsilon, 3}^{2}\right),
$$

which yields that

$$
I_{11}^{\varepsilon} \leq-\frac{\varepsilon^{2}}{2} \frac{d}{d t} \int \frac{1}{n^{0}+\varepsilon n^{1}}\left|\partial^{\gamma} \Delta \phi^{1}\right|^{2}+C\left(1+\varepsilon^{2} \|\left.\left|\left(\mathbf{u}^{1}, \phi^{1}\right)\right|\right|_{\varepsilon, 3} ^{2}\right) \|\left.\left|\phi^{1}\right|\right|_{\varepsilon, s} ^{2} .
$$

- Estimate of $I_{12}^{\varepsilon}$.

For the second term $I_{12}^{\varepsilon}$, we have

$$
\begin{aligned}
I_{12}^{\varepsilon} & =\int \frac{\varepsilon \partial^{\gamma} \Delta \phi^{1}}{n^{0}+\varepsilon n^{1}} \partial^{\gamma}\left(\partial_{t} n^{0} \phi^{1}\right)+\int \frac{\varepsilon \partial^{\gamma} \Delta \phi^{1}}{n^{0}+\varepsilon n^{1}} \partial^{\gamma}\left(n^{0} \partial_{t} \phi^{1}\right) \\
& =\int \frac{\varepsilon \partial^{\gamma} \Delta \phi^{1}}{n^{0}+\varepsilon n^{1}} \partial^{\gamma}\left(\partial_{t} n^{0} \phi^{1}\right)+\int \frac{\varepsilon \partial^{\gamma} \Delta \phi^{1}}{n^{0}+\varepsilon n^{1}} n^{0} \partial_{t} \partial^{\gamma} \phi^{1}+\int \frac{\varepsilon \partial^{\gamma} \Delta \phi^{1}}{n^{0}+\varepsilon n^{1}}\left[\partial^{\gamma}, n^{0}\right] \partial_{t} \phi^{1} \\
& =: \sum_{i=1}^{3} I_{12 i}^{\varepsilon} .
\end{aligned}
$$

For the first term $I_{121}^{\varepsilon}$, by the multiplicative estimates (A.1), we have

$$
\begin{aligned}
\left\|\partial^{\gamma}\left(\partial_{t} n^{0} \phi^{1}\right)\right\|_{L^{2}} & \leq C\left(\left\|\partial_{t} n^{0}\right\|_{L^{\infty}}\left\|\phi^{1}\right\|_{H^{s}}+\left\|\partial_{t} n^{0}\right\|_{H^{s}}\left\|\phi^{1}\right\|_{L^{\infty}}\right) \\
& \leq C\left(\left\|\phi^{1}\right\|_{H^{3}}+\left\|\phi^{1}\right\|_{H^{s}}\right)
\end{aligned}
$$

which yields

$$
\begin{aligned}
I_{121}^{\varepsilon} & \leq C\left(\left\|\phi^{1}\right\|_{H^{3}}+\left\|\phi^{1}\right\|_{H^{s}}\right)\left\|\varepsilon \Delta \phi^{1}\right\|_{H^{s}} \\
& \leq C\left(\left\|\phi^{1}\right\|_{H^{3}}^{2}+\left\|\phi^{1}\right\|_{\varepsilon, s}^{2}\right) .
\end{aligned}
$$


For the second term $I_{122}^{\varepsilon}$, we have by integration by parts,

$$
\begin{aligned}
I_{122}^{\varepsilon}= & -\int \nabla\left(\frac{n^{0}}{n^{0}+\varepsilon n^{1}}\right) \varepsilon \partial^{\gamma} \nabla \phi^{1} \partial_{t} \partial^{\gamma} \phi^{1}-\int\left(\frac{n^{0}}{n^{0}+\varepsilon n^{1}}\right) \varepsilon \partial^{\gamma} \nabla \phi^{1} \partial_{t} \nabla \partial^{\gamma} \phi^{1} \\
= & \int \nabla\left(\frac{n^{0}}{n^{\varepsilon}}\right) \varepsilon \partial^{\gamma+\gamma_{1}} \nabla \phi^{1} \partial_{t} \partial^{\gamma-\gamma_{1}} \phi^{1}+\int \nabla \partial^{\gamma_{1}}\left(\frac{n^{0}}{n^{\varepsilon}}\right) \varepsilon \partial^{\gamma} \nabla \phi^{1} \partial_{t} \partial^{\gamma-\gamma_{1}} \phi^{1} \\
& -\frac{\varepsilon}{2} \frac{d}{d t} \int \frac{n^{0}}{n^{\varepsilon}}\left|\partial^{\gamma} \nabla \phi^{1}\right|^{2}+\frac{\varepsilon}{2} \int \partial_{t}\left(\frac{n^{0}}{n^{\varepsilon}}\right)\left|\partial^{\gamma} \nabla \phi^{1}\right|^{2}=: I_{122 i}^{\varepsilon}
\end{aligned}
$$

where $\gamma_{1} \leq \gamma$ is a multiindex with $\left|\gamma_{1}\right|=1$. By Lemma 2.4 and Lemma 2.3, we have

$$
\begin{aligned}
\left\|\partial_{t} \partial^{\gamma-\gamma_{1}} \phi^{1}\right\|_{L^{2}}^{2} & \leq C\left(\left\|\partial_{t} \partial^{\gamma-\gamma_{1}} n^{1}\right\|^{2}+\left\|\phi^{1}\right\|_{H^{s-1}}^{2}\right) \\
& \leq C\left(1+\left\|\left(\mathbf{u}^{1}, \phi^{1}\right) \mid\right\|_{\varepsilon, s}^{2}\right),
\end{aligned}
$$

where $\gamma_{1} \leq \gamma,\left|\gamma_{1}\right|=1$ and hence $\left|\gamma-\gamma_{1}\right|=s-1$. On the other hand, by direct computation, we have

$$
\begin{aligned}
\left\|\nabla\left(\frac{n^{0}}{n^{\varepsilon}}\right)\right\|_{L^{\infty}} & \leq C\left(1+\varepsilon\left\|\nabla n^{1}\right\|_{L^{\infty}}\right) \leq C\left(1+\varepsilon^{2}\left\|\phi^{1}\right\| \|_{\varepsilon, 3}^{2}\right), \\
\left\|\nabla \partial^{\gamma_{1}}\left(\frac{n^{0}}{n^{\varepsilon}}\right)\right\|_{L^{\infty}} & \leq C\left(1+\varepsilon^{2}\left\|\nabla n^{1}\right\|_{L^{\infty}}^{2}+\varepsilon\left\|\nabla \partial^{\gamma_{1}} \phi^{1}\right\|_{L^{\infty}}\right) \\
& \leq C\left(1+\varepsilon^{2}\left\|\mid \phi^{1}\right\|_{\varepsilon, 4}^{2}\right),
\end{aligned}
$$

where we have used Lemma 2.2 and Sobolev embedding. Hence, by Hölder inequality, we obtain

$$
\begin{aligned}
I_{1221}^{\varepsilon}, I_{1222}^{\varepsilon} \leq & C\left\|\nabla\left(\frac{n^{0}}{n^{\varepsilon}}\right)\right\|_{L^{\infty}}\left\|\varepsilon \partial^{\gamma+\gamma_{1}} \nabla \phi^{1}\right\|_{L^{2}}\left\|\partial_{t} \partial^{\gamma-\gamma_{1}} \phi^{1}\right\|_{L^{2}} \\
& +C\left\|\nabla \partial^{\gamma_{1}}\left(\frac{n^{0}}{n^{\varepsilon}}\right)\right\|_{L^{\infty}}\left\|\varepsilon \partial^{\gamma} \nabla \phi^{1}\right\|_{L^{2}}\left\|\partial_{t} \partial^{\gamma-\gamma_{1}} \phi^{1}\right\|_{L^{2}} \\
\leq & C\left(1+\varepsilon^{2}\|\| \phi^{1} \|_{\varepsilon, 4}^{2}\right)\left(1+\|\|\left(\mathbf{u}^{1}, \phi^{1}\right)\|\|_{\varepsilon, s}^{2}\right),
\end{aligned}
$$

where we have used the boundedness of the Riesz operator. Similarly,

$$
\left\|\partial_{t}\left(\frac{n^{0}}{n^{\varepsilon}}\right)\right\|_{L^{\infty}} \leq C\left(1+\varepsilon^{2}\left\|\partial_{t} n^{1}\right\|_{L^{\infty}}^{2}\right) \leq C\left(1+\varepsilon^{2}\|\|\left(\mathbf{u}^{1}, \phi^{1}\right) \|_{\varepsilon, 3}^{2}\right),
$$

which yields that

$$
I_{1224}^{\varepsilon} \leq C\left(1+\varepsilon^{2}\left\|\left|\left(\mathbf{u}^{1}, \phi^{1}\right)\right|\right\|_{\varepsilon, 3}^{2}\right)\left\|\left|\phi^{1}\right|\right\|_{\varepsilon, s}^{2} .
$$

Therefore, $I_{122}^{\varepsilon}$ is bounded by

$$
I_{122}^{\varepsilon} \leq-\frac{\varepsilon}{2} \frac{d}{d t} \int \frac{n^{0}}{n^{\varepsilon}}\left|\partial^{\gamma} \nabla \phi^{1}\right|^{2}+C\left(1+\varepsilon^{2}\left|\left\|\left(\mathbf{u}^{1}, \phi^{1}\right) \mid\right\|_{\varepsilon, 4}^{2}\right)\left(1+\left|\left\|\left(\mathbf{u}^{1}, \phi^{1}\right)|\||_{\varepsilon, s}^{2}\right) .\right.\right.\right.
$$

For the third term $I_{123}^{\varepsilon}$, we have

$$
\begin{aligned}
I_{123}^{\varepsilon} & \leq C \varepsilon^{2}\left\|\Delta \phi^{1}\right\|_{H^{s}}^{2}+C\left\|\left[\partial^{\gamma}, n^{0}\right] \partial_{t} \phi^{1}\right\|_{L^{2}}^{2} \\
& \leq C \varepsilon^{2}\left\|\Delta \phi^{1}\right\|_{H^{s}}^{2}+C\left(\left\|\nabla n^{0}\right\|_{L^{\infty}}^{2}\left\|\partial_{t} \phi^{1}\right\|_{H^{s-1}}^{2}+\left\|\partial_{t} \phi^{1}\right\|_{L^{\infty}}^{2}\left\|n^{0}\right\|_{H^{s}}^{2}\right) \\
& \leq C\left(1+\|\|\left(\mathbf{u}^{1}, \phi^{1}\right)\|\|_{\varepsilon, s}^{2}+\|\|\left(\mathbf{u}^{1}, \phi^{1}\right) \|_{\varepsilon, 3}^{2}\right),
\end{aligned}
$$


thanks to Lemma A.1 in the second inequality and Lemma 2.4 and Lemma 2.3 in the last inequality. In summary, $I_{12}^{\varepsilon}$ can be bounded by

$$
\begin{aligned}
I_{12}^{\varepsilon} \leq & -\frac{\varepsilon}{2} \frac{d}{d t} \int \frac{n^{0}}{n^{\varepsilon}}\left|\partial^{\gamma} \nabla \phi^{1}\right|^{2} \\
& +C\left(1+\varepsilon^{2}\left|\left\|\left(\mathbf{u}^{1}, \phi^{1}\right) \mid\right\|_{\varepsilon, 4}^{2}\right)\left(1+\left\|||\left(\mathbf{u}^{1}, \phi^{1}\right)\left|\left\|_{\varepsilon, s}^{2}+\right\|\right|\left(\mathbf{u}^{1}, \phi^{1}\right) \mid\right\|_{\varepsilon, 3}^{2}\right) .\right.
\end{aligned}
$$

- Estimate of $I_{13}^{\varepsilon}$.

For the third term $I_{13}^{\varepsilon}$, it is straightforward that

$$
I_{13}^{\varepsilon} \leq C\left(1+\varepsilon^{2}\left\|\Delta \phi^{1}\right\|_{H^{s}}^{2}\right) .
$$

- Estimate of $I_{14}^{\varepsilon}$.

For the fourth term $I_{14}^{\varepsilon}$, we have

$$
\begin{aligned}
\left|I_{14}^{\varepsilon}\right| & \leq C \varepsilon^{2}\left\|\Delta \phi^{1}\right\|_{H^{s}}^{2}+C \varepsilon\left\|\partial_{t} R^{1}\right\|_{H^{s}}^{2} \\
& \leq C \varepsilon^{2}\left\|\Delta \phi^{1}\right\|_{H^{s}}^{2}+C_{1}\left(\left\|\phi^{1}\right\|_{H^{s}}^{2}+\varepsilon\left\|\partial_{t} \phi^{1}\right\|_{H^{s}}^{2}\right) \\
& \leq C \varepsilon^{2}\left\|\Delta \phi^{1}\right\|_{H^{s}}^{2}+C_{1}\left(\left\|\phi^{1}\right\|_{H^{s}}^{2}+\left\|\partial_{t} n^{1}\right\|_{H^{s-1}}^{2}\right) \\
& \leq C\left(1+\|\|\left(\mathbf{u}^{1}, \phi^{1}\right)\|\|_{\varepsilon, s}^{2}\right),
\end{aligned}
$$

where we have used Hölder inequality in the first inequality, Lemma 2.1 in the second inequality, Lemma 2.4 in the third inequality and Lemma 2.3 in the last inequality. Here, we also have used the fact that $\left\|\partial_{t} \phi^{1}\right\|_{H^{s}} \approx\left\|\partial_{t} \phi^{1}\right\|_{H^{s-1}}+\left\|\partial_{t} \partial^{\alpha} \nabla \phi^{1}\right\|_{L^{2}}$ with $|\gamma|=s-1$ and $\left\|\phi^{1}\right\|_{H^{s-1}} \leq\left\|\phi^{1}\right\|_{H^{s}}$ for all integers $s>0$. $I^{\varepsilon}$.

By (2.51), using (2.52), (2.53), 2.54) and (2.55), we obtain the estimate (2.50) for

Now, we can end the proof of Lemma 2.11

End of proof of Lemma 2.11, By using (2.40), the estimates of (2.41), (2.42), (2.43) and (2.45), and Lemmas 2.12 and 2.13, we close the proof of Lemma 2.11.

Summarizing these lemmas, we obtain the following.

Proposition 2.14. Let $s \geq 0$ be a positive integer and $\left(n^{1}, \mathbf{u}^{1}, \phi^{1}\right)$ a smooth solution for the system (2.2). There exists $\varepsilon_{1}>0$ and $C, C^{\prime}>0$ such that for any $0<\varepsilon<\varepsilon_{1}$, there holds

$$
\begin{aligned}
& \varepsilon\left\|\nabla \mathbf{u}^{1}(t)\right\|_{H^{s}}^{2}+\varepsilon\left\|\nabla \phi^{1}(t)\right\|_{H^{s}}^{2}+\varepsilon^{2}\left\|\Delta \phi^{1}(t)\right\|_{H^{s}}^{2} \\
& \leq C^{\prime}\left(\varepsilon\left\|\nabla \mathbf{u}^{1}(0)\right\|_{H^{s}}^{2}+\varepsilon\left\|\nabla \phi^{1}(0)\right\|_{H^{s}}^{2}+\varepsilon^{2}\left\|\Delta \phi^{1}(0)\right\|_{H^{s}}^{2}\right) \\
& \quad+C \int_{0}^{t}\left(1+\varepsilon^{2}\|\|\left(\mathbf{u}^{1}, \phi^{1}\right)\|\|_{\varepsilon, 4}^{2}\right)\left(1+\|\|\left(\mathbf{u}^{1}, \phi^{1}\right)\|\|_{\varepsilon, s \vee 3}^{2}\right) d \tau
\end{aligned}
$$

where $C^{\prime}$ depends only on $\sigma^{\prime}$ and $\sigma^{\prime \prime}$.

Proof. This is shown by integrating (2.39) over $[0, t]$ and summing them up for $|\gamma| \leq s$, and then using $\sigma^{\prime}<n^{0}<\sigma^{\prime \prime}$ and $\sigma^{\prime} / 2<n^{\varepsilon}<2 \sigma^{\prime \prime}$ for any $t \in\left[0, T_{\varepsilon}\right]$ in (1.4) and (2.5) for $0<\varepsilon<\varepsilon_{1}$.

2.5. End of proof of Theorem 1.3. Now, we are in a good position to end the proof of Theorem 1.3 . 
Proof. Let $s \geq 7$ be an integer. By Proposition 2.10 and Proposition 2.14 and recalling the definition of the norm (1.7), we obtain the Gronwall type inequality

$$
\begin{aligned}
\left\|\left|\left(\mathbf{u}^{1}, \phi^{1}\right)(t) \|\right|_{\varepsilon, s}^{2} \leq\right. & C^{\prime} C_{\varepsilon}(0) \\
& +C \int_{0}^{t}\left(1+\varepsilon^{3}\left\|\left|\left(\mathbf{u}^{1}, \phi^{1}\right) \|\right|_{\varepsilon, 5}^{3}\right)\left(1+\left\|\mid\left(\mathbf{u}^{1}, \phi^{1}\right)\right\| \|_{\varepsilon, s}^{2}\right) d \tau\right.
\end{aligned}
$$

where $C_{\varepsilon}(0)=\|||\left(\mathbf{u}^{1}, \phi^{1}\right)(0)||_{\varepsilon, s}^{2}$ and we have used the fact that $\||\cdot|\|_{\varepsilon, 3} \leq\||\cdot|\|_{\varepsilon, s}$ for $s \geq 3$. From (2.4), there exists $\varepsilon_{1}>0$ such that for any $0<\varepsilon<\varepsilon_{1}, \varepsilon^{3}\left\|\mid\left(\mathbf{u}^{1}, \phi^{1}\right)\right\| \|_{\varepsilon, 5}^{3} \leq 1$, and hence (2.57) yields

$$
\left.\left\|||\left(\mathbf{u}^{1}, \phi^{1}\right)(t)\right\|\right|_{\varepsilon, s} ^{2} \leq C_{2} C_{\varepsilon}(0)+C_{2} \int_{0}^{t}\left(1+\|\left.\left|\left(\mathbf{u}^{1}, \phi^{1}\right)\right|\right|_{\varepsilon, s} ^{2}\right) d \tau,
$$

where $C_{2}=\max \left\{C^{\prime}, 2 C\right\}$. On the other hand, from Lemma 2.2, there exists constant $C_{3}>1$ such that

$$
\left\|n^{1}(t)\right\|_{H^{s}}^{2} \leq C_{3}\left(1+\left\|\left|\phi^{1}\right|\right\|_{\varepsilon, s}^{2}\right) .
$$

Let $C_{0}=\sup _{0<\varepsilon<1} C_{\varepsilon}(0)$. We let $\tilde{C}$ in (2.4) satisfy $\tilde{C} \geq 2 C_{3}\left(1+C_{2} C_{0}\right) e^{C_{2} T_{\varepsilon}}$. Then from (2.58)

and hence from (2.59)

$$
\left\|\mid\left(\mathbf{u}^{1}, \phi^{1}\right)\right\| \|_{\varepsilon, s}^{2} \leq\left(1+C_{2} C_{0}\right) e^{C_{2} T_{\varepsilon}} \leq \tilde{C},
$$

$$
\left\|n^{1}(t)\right\|_{H^{s}}^{2} \leq C_{3}\left(1+\left(1+C_{2} C_{0}\right) e^{C_{2} T_{\varepsilon}}\right) \leq \tilde{C} .
$$

Then by the continuity principle, it is standard to get the uniform in $\varepsilon$ estimates for $\left\|\mid\left(n^{1}, \mathbf{u}^{1}, \phi^{1}\right)\right\|_{\varepsilon, s}$. In particular, for every $T^{\prime}<T, \varepsilon^{-1}\left(n^{\varepsilon}-n^{0}\right)$ and $\varepsilon^{-1}\left(\mathbf{u}^{\varepsilon}-\mathbf{u}^{0}\right)$ are bounded in $L^{\infty}\left(\left[0, T^{\prime}\right] ; H^{s}\right)$ and $L^{\infty}\left(\left[0, T^{\prime}\right] ; \mathbf{H}^{s}\right)$, respectively, uniformly in $\varepsilon$ for $\varepsilon$ small enough for some $s<s^{\prime}$.

3. Proof of Theorem 1.4. This section is devoted to the proof of Theorem 1.4. Let $\left(n^{\varepsilon, T_{i}}, \mathbf{u}^{\varepsilon, T_{i}}, \phi^{\varepsilon, T_{i}}\right)$ be a solution of (1.5) and $\left(n^{0, T_{i}}, \mathbf{u}^{0, T_{i}}, \phi^{0, T_{i}}\right)$ be a solution of (1.6) with the same initial data. Let

$$
n^{\varepsilon, T_{i}}=n^{0, T_{i}}+\varepsilon n^{1, T_{i}}, \quad \mathbf{u}^{\varepsilon, T_{i}}=\mathbf{u}^{0, T_{i}}+\varepsilon \mathbf{u}^{1, T_{i}}, \quad \phi^{\varepsilon, T_{i}}=\phi^{0, T_{i}}+\varepsilon \phi^{1, T_{i}} .
$$

Then $\left(n^{1, T_{i}}, \mathbf{u}^{1, T_{i}}, \phi^{1, T_{i}}\right)$ satisfy the remainder system:

$$
\left(R_{\varepsilon}\right)\left\{\begin{array}{c}
\partial_{t} n^{1, T_{i}}+\nabla \cdot\left(n^{0, T_{i}} \mathbf{u}^{1, T_{i}}+\mathbf{u}^{0, T_{i}} n^{1, T_{i}}\right)+\varepsilon \nabla \cdot\left(n^{1, T_{i}} \mathbf{u}^{1, T_{i}}\right)=0 \\
\partial_{t} \mathbf{u}^{1, T_{i}}+\mathbf{u}^{0, T_{i}} \cdot \nabla \mathbf{u}^{1, T_{i}}+\mathbf{u}^{1, T_{i}} \cdot \nabla \mathbf{u}^{0, T_{i}}+\varepsilon \mathbf{u}^{1, T_{i}} \cdot \nabla \mathbf{u}^{1, T_{i}} \\
+\frac{T_{i} \nabla n^{1, T_{i}}}{n^{0, T_{i}}+\varepsilon n^{1, T_{i}}}-\frac{T_{i} n^{1, T_{i}} \nabla n^{0, T_{i}}}{n^{0, T_{i}}\left(n^{0, T_{i}}+\varepsilon n^{1, T_{i}}\right)}=-\nabla \phi^{1, T_{i}} \\
-\varepsilon \Delta \phi^{1, T_{i}}=\Delta \phi^{0, T_{i}}+n^{1, T_{i}}-n^{0, T_{i}} \phi^{1, T_{i}}+\sqrt{\varepsilon} R^{1},
\end{array}\right.
$$

where $R^{1}$ is given in (2.3).

We need only show estimates uniform in $\varepsilon$ and $T_{i}$ for $\left(n^{1, T_{i}}, \mathbf{u}^{1, T_{i}}\right)$. We also let $\tilde{C}$ be a constant to be determined later, much larger than the bound of $\left\|\left(n_{0}, \mathbf{u}_{0}\right)\right\|_{H^{s}}$, such that on $\left[0, T^{\varepsilon, T_{i}}\right]$ :

$$
\sup _{\left[0, T^{\varepsilon, T_{i}}\right]}\left\|\left(n^{1, T_{i}}, \mathbf{u}^{1, T_{i}}, \phi^{1, T_{i}}\right)\right\|_{H^{s}} \leq \tilde{C} .
$$


From the expression (1), there exists some $\varepsilon_{1}=\varepsilon_{1}(\tilde{C})>0$ and $T_{i 1}>0$ such that $\sigma^{\prime} / 2<n^{\varepsilon, T_{i}}<2 \sigma^{\prime}$ and $\left|\mathbf{u}^{\varepsilon, T_{i}}\right| \leq 1 / 2$ on $\left[0, T^{\varepsilon, T_{i}}\right]$ for all $0<\varepsilon<\varepsilon_{1}$ and $0<T_{i}<T_{i 1}$.

Let $\gamma$ be a multiindex with $|\gamma|=s$. By taking $\partial^{\gamma}$ to $(3.2 \mathrm{~b})$, and then taking the $L^{2}$ inner product with $\partial^{\gamma} \mathbf{u}^{1, T_{i}}$, we obtain an equality similar to (2.12) but with the following two more terms on the RHS:

$$
V+V I:=-T_{i} \int \partial^{\gamma} \mathbf{u}^{1, T_{i}} \partial^{\gamma}\left(\frac{\nabla n^{1, T_{i}}}{n^{0, T_{i}}+\varepsilon n^{1, T_{i}}}\right)+T_{i} \int \partial^{\gamma} \mathbf{u}^{1, T_{i}} \partial^{\gamma}\left(\frac{\nabla n^{0, T_{i}}}{n^{0, T_{i}}} \frac{n^{1}}{n^{0, T_{i}}+\varepsilon n^{1, T_{i}}}\right) .
$$

- Estimate of the term VI.

For this term, we obtain by using the Leibnitz formula and multiplicative estimates

$$
V I \leq T_{i} C(\varepsilon \tilde{C})\left\|\left(\partial^{\gamma} \mathbf{u}^{1, T_{i}}, \partial^{\gamma} n^{1, T_{i}}\right)\right\|^{2},
$$

where $\tilde{C}$ is given in (2.4).

- Estimate of the term $V$.

For this term, we rewrite

$$
\begin{aligned}
V= & -T_{i} \int \frac{\partial^{\gamma} \mathbf{u}^{1, T_{i}} \cdot \nabla \partial^{\gamma} n^{1, T_{i}}}{n^{0, T_{i}}+\varepsilon n^{1, T_{i}}}-T_{i} \int \partial^{\gamma} \mathbf{u}^{1, T_{i}} \cdot\left[\partial^{\gamma}, \frac{1}{n^{0, T_{i}}+\varepsilon n^{1, T_{i}}}\right] \nabla n^{1, T_{i}} \\
= & T_{i} \int \frac{\partial^{\gamma} \nabla \cdot \mathbf{u}^{1, T_{i}} \partial^{\gamma} n^{1, T_{i}}}{n^{0, T_{i}}+\varepsilon n^{1, T_{i}}}+T_{i} \int \partial^{\gamma} \mathbf{u}^{1, T_{i}} \partial^{\gamma} n^{1, T_{i}} \cdot \nabla\left(\frac{1}{n^{0, T_{i}}+\varepsilon n^{1, T_{i}}}\right) \\
& -T_{i} \int \partial^{\gamma} \mathbf{u}^{1, T_{i}} \cdot\left[\partial^{\gamma}, \frac{1}{n^{0, T_{i}}+\varepsilon n^{1, T_{i}}}\right] \nabla n^{1, T_{i}}=: V_{1}+V_{2}+V_{3} .
\end{aligned}
$$

For $V_{2}$ and $V_{3}$, we obtain by using commutator estimates (A.1) that

$$
V_{2}+V_{3} \leq T_{i} C(\varepsilon \tilde{C})\left\|\left(\partial^{\gamma} \mathbf{u}^{1, T_{i}}, \partial^{\gamma} n^{1, T_{i}}\right)\right\|^{2} .
$$

Recalling decomposition (2.17), we decompose $V_{1}$ into

$$
\begin{aligned}
V_{1}= & -T_{i} \int \frac{\partial_{t} \partial^{\gamma} n^{1, T_{i}} \partial^{\gamma} n^{1, T_{i}}}{\left(n^{0, T_{i}}+\varepsilon n^{1, T_{i}}\right)^{2}}-T_{i} \int \frac{\left[\partial^{\gamma}, n^{0, T_{i}}+\varepsilon n^{1, T_{i}}\right] \nabla \cdot \mathbf{u}^{1, T_{i}} \partial^{\gamma} n^{1, T_{i}}}{\left(n^{0, T_{i}}+\varepsilon n^{1, T_{i}}\right)^{2}} \\
& -T_{i} \int \frac{\partial^{\gamma}\left(\left(\mathbf{u}^{0, T_{i}}+\varepsilon \mathbf{u}^{1, T_{i}}\right) \cdot \nabla n^{1, T_{i}}\right) \partial^{\gamma} n^{1, T_{i}}}{\left(n^{0, T_{i}}+\varepsilon n^{1, T_{i}}\right)^{2}}-T_{i} \int \frac{\partial^{\gamma}\left(\mathbf{u}^{1, T_{i}} \cdot \nabla n^{0, T_{i}}\right) \partial^{\gamma} n^{1, T_{i}}}{\left(n^{0, T_{i}}+\varepsilon n^{1, T_{i}}\right)^{2}} \\
& -T_{i} \int \frac{\partial^{\gamma}\left(n^{1, T_{i}} \nabla \cdot \mathbf{u}^{0, T_{i}}\right) \partial^{\gamma} n^{1, T_{i}}}{\left(n^{0, T_{i}}+\varepsilon n^{1, T_{i}}\right)^{2}}=: V_{11}+\cdots+V_{15} .
\end{aligned}
$$

The term $V_{11}$ can be estimated as

$$
V_{11}=-\frac{T_{i}}{2} \frac{d}{d t} \int \frac{\left|\partial^{\gamma} n^{1, T_{i}}\right|^{2}}{\left(n^{0, T_{i}}+\varepsilon n^{1, T_{i}}\right)^{2}} .
$$

The estimate of term $V_{12}$ is similar to $I_{3}$ in (2.18), and the estimate of term $V_{13}$ is similar to $I I$ in (2.12). After integration by parts and commutator estimates, they can be estimated as

$$
V_{12}+V_{13} \leq T_{i} C(\varepsilon \tilde{C})\left\|\left(n^{1, T_{i}}, \mathbf{u}^{1, T_{i}}\right)\right\|_{H^{s}}^{2} .
$$

The estimates of the last two terms $V_{14}$ and $V_{15}$ are direct since the derivative on $n^{1, T_{i}}$ and $\mathbf{u}^{1, T_{i}}$ is no greater than $|\gamma|$ and we have

$$
V_{14}+V_{15} \leq T_{i} C(\varepsilon \tilde{C})\left\|\left(n^{1, T_{i}}, \mathbf{u}^{1, T_{i}}\right)\right\|_{H^{s}}^{2} .
$$


These estimates lead to the following:

$$
V+V I \leq-\frac{T_{i}}{2} \frac{d}{d t} \int \frac{\left|\partial^{\gamma} n^{1, T_{i}}\right|^{2}}{\left(n^{0, T_{i}}+\varepsilon n^{1, T_{i}}\right)^{2}}+T_{i} C(\varepsilon \tilde{C})\left\|\left(n^{1, T_{i}}, \mathbf{u}^{1, T_{i}}\right)\right\|_{H^{s}}^{2} .
$$

Therefore, similar to Proposition 2.10, there exist $\varepsilon_{1}>0$ and $T_{i 1}>0$ and constants $C^{\prime}, C>0$ such that

$$
\begin{aligned}
& T_{i}\left\|n^{1, T_{i}}(t)\right\|_{H^{s}}^{2}+\left\|\mathbf{u}^{1, T_{i}}(t)\right\|_{H^{s}}^{2}+\left\|\phi^{1, T_{i}}(t)\right\|_{H^{s}}^{2}+\varepsilon\left\|\nabla \phi^{1, T_{i}}(t)\right\|_{H^{s}}^{2} \\
& \leq C^{\prime}\left(T_{i}\left\|n^{1, T_{i}}(0)\right\|_{H^{s}}^{2}+\left\|\mathbf{u}^{1, T_{i}}(0)\right\|_{H^{s}}^{2}+\left\|\phi^{1, T_{i}}(0)\right\|_{H^{s}}^{2}+\varepsilon\left\|\nabla \phi^{1, T_{i}}(0)\right\|_{H^{s}}^{2}\right) \\
& \quad+C \int_{0}^{t} T_{i} C(\varepsilon \tilde{C})\left\|\left(n^{1, T_{i}}, \mathbf{u}^{1, T_{i}}\right)\right\|_{H^{s}}^{2} \\
& \quad+\left(1+\varepsilon^{3}\|\|\left(\mathbf{u}^{1, T_{i}}, \phi^{1, T_{i}}\right) \|_{\varepsilon, 5}^{3}\right)\left(1+\|\|\left(\mathbf{u}^{1, T_{i}}, \phi^{1, T_{i}}\right)\|\|_{\varepsilon, s \vee 3}^{2}\right) d \tau,
\end{aligned}
$$

where $C^{\prime}$ depends only on $\sigma^{\prime}$ and $\sigma^{\prime \prime}$.

Likewise, at the weighted $(s+1)$-order, there holds

$$
\begin{aligned}
& T_{i} \varepsilon\left\|\nabla n^{1, T_{i}}(t)\right\|_{H^{s}}^{2}+\varepsilon\left\|\nabla \mathbf{u}^{1, T_{i}}(t)\right\|_{H^{s}}^{2}+\varepsilon\left\|\nabla \phi^{1, T_{i}}(t)\right\|_{H^{s}}^{2}+\varepsilon^{2}\left\|\Delta \phi^{1, T_{i}}(t)\right\|_{H^{s}}^{2} \\
& \leq C^{\prime}\left(T_{i} \varepsilon\left\|\nabla n^{1, T_{i}}(0)\right\|_{H^{s}}^{2}+\varepsilon\left\|\nabla \mathbf{u}^{1, T_{i}}(0)\right\|_{H^{s}}^{2}+\varepsilon\left\|\nabla \phi^{1, T_{i}}(0)\right\|_{H^{s}}^{2}+\varepsilon^{2}\left\|\Delta \phi^{1, T_{i}}(0)\right\|_{H^{s}}^{2}\right) \\
& +C \int_{0}^{t} T_{i} \varepsilon C(\varepsilon \tilde{C})\left\|\left(n^{1, T_{i}}, \mathbf{u}^{1, T_{i}}\right)\right\|_{H^{s+1}}^{2} \\
& \quad+\left(1+\varepsilon^{2}\left\|\left(\mathbf{u}^{1, T_{i}}, \phi^{1, T_{i}}\right)\right\|_{\varepsilon, 4}^{2}\right)\left(1+\left\|\left(\mathbf{u}^{1, T_{i}}, \phi^{1, T_{i}}\right)\right\| \|_{\varepsilon, s \vee 3}^{2}\right) d \tau,
\end{aligned}
$$

where $C^{\prime}$ depends only on $\sigma^{\prime}$ and $\sigma^{\prime \prime}$.

Now let $\left\|\left|n^{1, T_{i}}\right|\right\|_{\varepsilon, s}^{2}=\left\|n^{1, T_{i}}\right\|_{H^{s}}^{2}+\varepsilon\left\|\nabla n^{1, T_{i}}\right\|_{H^{s}}^{2}$. Putting (3.6) and (3.7) together, we then obtain the following Gronwall type inequality:

$$
\begin{aligned}
& \|\|\left(\sqrt{T_{i}} n^{1, T_{i}}, \mathbf{u}^{1, T_{i}}, \phi^{1, T_{i}}\right)(t) \|\left.\right|_{\varepsilon, s} ^{2} \leq C^{\prime} C_{\varepsilon}(0) \\
& \quad+\int_{0}^{t} C(\varepsilon \tilde{C})\left(1+\varepsilon^{3}\left\|\mid\left(\mathbf{u}^{1, T_{i}}, \phi^{1, T_{i}}\right)\right\|_{\varepsilon, 5}^{3}\right)\left(1+\|\|\left(\sqrt{T_{i}} n^{1, T_{i}}, \mathbf{u}^{1, T_{i}}, \phi^{1, T_{i}}\right)\|\|_{\varepsilon, s}^{2}\right) d \tau
\end{aligned}
$$

where $C_{\varepsilon}(0)=\|\|\left(\mathbf{u}^{1, T_{i}}, \phi^{1, T_{i}}\right)(0) \mid \|_{\varepsilon, s}^{2}$ and $s \vee 3=s$ for $s \geq 3$. From (3.3), there exists $\varepsilon_{1}>0$ such that for any $0<\varepsilon<\varepsilon_{1}, \varepsilon^{3}\left\|\mid\left(\mathbf{u}^{1, T_{i}}, \phi^{1, T_{i}}\right)\right\| \|_{\varepsilon, 5}^{3} \leq 1$ and $C(\varepsilon \tilde{C}) \leq C(1)$, and hence (3.8) yields

$$
\begin{aligned}
\|\|\left(\sqrt{T_{i}} n^{1, T_{i}}, \mathbf{u}^{1, T_{i}}, \phi^{1, T_{i}}\right)(t)\|\|_{\varepsilon, s}^{2} \leq & C_{2} C_{\varepsilon}(0) \\
& +C_{2} \int_{0}^{t}\left(1+\left\|\mid\left(\sqrt{T_{i}} n^{1, T_{i}}, \mathbf{u}^{1, T_{i}}, \phi^{1, T_{i}}\right)\right\| \|_{\varepsilon, s}^{2}\right) d \tau,
\end{aligned}
$$

where $C_{2}=\max \left\{C^{\prime}, 2 C(1)\right\}$. Then invoking Lemma 2.2 and following the proof of Theorem 1.3 in Section 2.5. we obtain the estimates uniform both in $\varepsilon$ and $T_{i}$ for $\left\|\left(n^{1, T_{i}}, \mathbf{u}^{1, T_{i}}, \phi^{1, T_{i}}\right)\right\|_{H^{s}}$. In deed, we obtain that $\left\|n^{1, T_{i}}\right\|_{H^{s}}^{2}+T_{i} \varepsilon\left\|\nabla n^{1, T_{i}}\right\|_{H^{s}}^{2}$ is uniformly bounded. In particular, for every $T^{\prime}<T, \varepsilon^{-1}\left(n^{\varepsilon}-n^{0}\right)$ and $\varepsilon^{-1}\left(\mathbf{u}^{\varepsilon}-\mathbf{u}^{0}\right)$ are bounded in $L^{\infty}\left(\left[0, T^{\prime}\right] ; H^{s}\right)$ and $L^{\infty}\left(\left[0, T^{\prime}\right] ; \mathbf{H}^{s}\right)$, respectively, uniformly in $\varepsilon$ and $T_{i}$ for $\varepsilon$ small enough for some $s<s^{\prime}$. This completes the proof. 
Appendix A. Commutator estimates. For the reader's convenience, we give two important inequalities that are widely used throughout this paper [13, Lemma X1 and Lemma X4].

Lemma A.1. Let $\alpha$ be any multiindex with $|\alpha|=k$ and $p \in(1, \infty)$. Then there exists some constant $C>0$ such that

$$
\begin{aligned}
\left\|\partial_{x}^{\alpha}(f g)\right\|_{L^{p}} & \leq C\left\{\|f\|_{L^{p_{1}}}\|g\|_{\dot{H}^{k, p_{2}}}+\|f\|_{\dot{H}^{k, p_{3}}}\|g\|_{L^{p_{4}}}\right\}, \\
\left\|\left[\partial_{x}^{\alpha}, f\right] g\right\|_{L^{p}} & \leq C\left\{\|\nabla f\|_{L^{p_{1}}}\|g\|_{\dot{H}^{k-1, p_{2}}}+\|f\|_{\dot{H}^{k, p_{3}}}\|g\|_{L^{p^{4}}}\right\},
\end{aligned}
$$

where $f, g \in \mathcal{S}$, the Schwartz class and $p_{2}, p_{3} \in(1,+\infty)$ are such that

$$
\frac{1}{p}=\frac{1}{p_{1}}+\frac{1}{p_{2}}=\frac{1}{p_{3}}+\frac{1}{p_{4}}
$$

\section{REFERENCES}

[1] Y. Brenier, Convergence of the Vlasov-Poisson system to the incompressible Euler equations, Comm. Partial Differential Equations 25 (2000), no. 3-4, 737-754, DOI 10.1080/03605300008821529. MR.1748352 (2001c:76124)

[2] Stéphane Cordier and Emmanuel Grenier, Quasineutral limit of an Euler-Poisson system arising from plasma physics, Comm. Partial Differential Equations 25 (2000), no. 5-6, 1099-1113, DOI 10.1080/03605300008821542. MR.1759803 (2001c:82078)

[3] P. Degond, H. Liu, D. Savelief, and M.-H. Vignal, Numerical approximation of the Euler-PoissonBoltzmann model in the quasineutral limit, J. Sci. Comput. 51 (2012), no. 1, 59-86, DOI 10.1007/s10915-011-9495-1. MR 2891946

[4] David Gérard-Varet, Daniel Han-Kwan, and Frédéric Rousset, Quasineutral limit of the EulerPoisson system for ions in a domain with boundaries, Indiana Univ. Math. J. 62 (2013), no. 2, 359-402, DOI 10.1512/iumj.2013.62.4900. MR3158514

[5] E. Grenier, Oscillatory perturbations of the Navier-Stokes equations, J. Math. Pures Appl. (9) 76 (1997), no. 6, 477-498, DOI 10.1016/S0021-7824(97)89959-X. MR1465607 (98h:35189)

[6] E. Grenier, Pseudo-differential energy estimates of singular perturbations, Comm. Pure Appl. Math. 50 (1997), no. 9, 821-865, DOI 10.1002/(SICI)1097-0312(199709)50:9〈821::AID-CPA2〉3.3.CO;2-3. MR 1459589 (98g:35014)

[7] Y. Guo, A.D. Ionescu and B. Pausader, Global solutions of the Euler-Maxwell two-fluid system in 3D. arXiv: 1303.1060v1.

[8] Yan Guo and Benoit Pausader, Global smooth ion dynamics in the Euler-Poisson system, Comm. Math. Phys. 303 (2011), no. 1, 89-125, DOI 10.1007/s00220-011-1193-1. MR2775116(2012e:82075)

[9] Yan Guo and Xueke Pu, KdV limit of the Euler-Poisson system, Arch. Ration. Mech. Anal. 211 (2014), no. 2, 673-710, DOI 10.1007/s00205-013-0683-z. MR3149069

[10] Daniel Han-Kwan, Quasineutral limit of the Vlasov-Poisson system with massless electrons, Comm. Partial Differential Equations 36 (2011), no. 8, 1385-1425, DOI 10.1080/03605302.2011.555804. MR:2825596 (2012g:35349)

[11] Daniel Han-Kwan, From Vlasov-Poisson to Korteweg-de Vries and Zakharov-Kuznetsov, Comm. Math. Phys. 324 (2013), no. 3, 961-993, DOI 10.1007/s00220-013-1825-8. MR3123542

[12] Song Jiang, QiangChang Ju, HaiLiang Li, and Yong Li, Quasi-neutral limit of the full bipolar EulerPoisson system, Sci. China Math. 53 (2010), no. 12, 3099-3114, DOI 10.1007/s11425-010-4114-4. MR:2746309 (2011h:35223)

[13] Tosio Kato and Gustavo Ponce, Commutator estimates and the Euler and Navier-Stokes equations, Comm. Pure Appl. Math. 41 (1988), no. 7, 891-907, DOI 10.1002/cpa.3160410704. MR951744 (90f:35162)

[14] N. Krall and A. Trivelpiece, Principles of plasma physics, San Francisco Press, 1986.

[15] David Lannes, Felipe Linares, and Jean-Claude Saut, The Cauchy problem for the Euler-Poisson system and derivation of the Zakharov-Kuznetsov equation, Studies in phase space analysis with applications to PDEs, Progr. Nonlinear Differential Equations Appl., vol. 84, Birkhäuser/Springer, New York, 2013, pp. 181-213, DOI 10.1007/978-1-4614-6348-1_10. MR3185896 
[16] Grégoire Loeper, Quasi-neutral limit of the Euler-Poisson and Euler-Monge-Ampère systems, Comm. Partial Differential Equations 30 (2005), no. 7-9, 1141-1167, DOI 10.1080/03605300500257545. MR2180297 (2007b:35267)

[17] A. Majda, Compressible fluid flow and systems of conservation laws in several space variables, Applied Mathematical Sciences, vol. 53, Springer-Verlag, New York, 1984. MR748308 (85e:35077)

[18] Yue-Jun Peng and Shu Wang, Convergence of compressible Euler-Maxwell equations to incompressible Euler equations, Comm. Partial Differential Equations 33 (2008), no. 1-3, 349-376, DOI 10.1080/03605300701318989. MR2398233 (2009b:35339)

[19] Xueke $\mathrm{Pu}$, Dispersive limit of the Euler-Poisson system in higher dimensions, SIAM J. Math. Anal. 45 (2013), no. 2, 834-878, DOI 10.1137/120875648. MR.3045650

[20] Xueke $\mathrm{Pu}$, Quasineutral limit of the pressureless Euler-Poisson equation, Appl. Math. Lett. 30 (2014), 33-37, DOI 10.1016/j.aml.2013.12.008. MR3162389

[21] M. Slemrod and N. Sternberg, Quasi-neutral limit for Euler-Poisson system, J. Nonlinear Sci. 11 (2001), no. 3, 193-209, DOI 10.1007/s00332-001-0004-9. MR.1852940 (2002f:35189)

[22] Shlomo Engelberg, Hailiang Liu, and Eitan Tadmor, Critical thresholds in Euler-Poisson equations, Special Issue dedicated to Professors Ciprian Foias and Roger Temam (Bloomington, IN, 2000), Indiana Univ. Math. J. 50 (2001), 109-157, DOI 10.1512/iumj.2001.50.2177. MR1855666 (2002i:35149)

[23] Elias M. Stein, Singular integrals and differentiability properties of functions, Princeton Mathematical Series, No. 30, Princeton University Press, Princeton, N.J., 1970. MR0290095 (44 \#7280)

[24] Shu Wang, Quasineutral limit of Euler-Poisson system with and without viscosity, Comm. Partial Differential Equations 29 (2004), no. 3-4, 419-456, DOI 10.1081/PDE-120030403. MR2041602 (2005i:35225)

[25] Shu Wang and Song Jiang, The convergence of the Navier-Stokes-Poisson system to the incompressible Euler equations, Comm. Partial Differential Equations 31 (2006), no. 4-6, 571-591, DOI 10.1080/03605300500361487. MR2233033 (2008f:35319) 